\title{
Exosomes From Human Umbilical Cord-derived Mesenchymal Stem Cells Alleviate Osteoarthritis by Balancing the Synthesis and Degradation of Cartilage Extracellular Matrix and Regulating Macrophage Polarization
}

\section{Pengdong Li}

Jilin University

\section{Shuang Lv}

Jilin University

\section{Wenyue Jiang}

The Sixth Affiliated Hospital of Guangzhou Medical University: Qingyuan People's Hospital

Lihui Si

Jilin University Second Hospital

\section{Baojian Liao}

The Sixth Affiliated Hospital of Guangzhou Medical University: Qingyuan People's Hospital

\section{Guifang Zhao}

The Sixth Affiliated Hospital of Guangzhou Medical University: Qingyuan People's Hospital

\section{Ziran Xu}

Jilin University

\section{Lina Wang}

Jilin University

Jia Zhang

The Sixth Affiliated Hospital of Guangzhou Medical University: Qingyuan People's Hospital

Haitao Wu

Jilin University

\section{Qian Peng}

The Sixth Affiliated Hospital of Guangzhou Medical University: Qingyuan People's Hospital

\section{Zhaohui Li}

The Sixth Affiliated Hospital of Guangzhou Medical University: Qingyuan People's Hospital Ling Qi

The Sixth Affiliated Hospital of Guangzhou Medical University: Qingyuan People's Hospital

Guangfan Chi ( $\square$ guangfan130@jlu.edu.cn )

Jilin University https://orcid.org/0000-0001-7096-9106 


\section{Yulin Li}

Jilin University

\section{Research Article}

Keywords: hUC-MSCs, exosome, osteoarthritis, chondrocyte, macrophage

Posted Date: June 17th, 2021

DOl: https://doi.org/10.21203/rs.3.rs-618845/v1

License: (c) (1) This work is licensed under a Creative Commons Attribution 4.0 International License. Read Full License 


\section{Abstract}

\section{Background}

Osteoarthritis $(\mathrm{OA})$ is one of the most common joint diseases and a major public health concern. Current therapies for OA can relieve symptoms but offer no potential for cartilage regeneration. Mesenchymal stem cells (MSCs) have been widely used for the treatment of OA owing to their paracrine secretion of trophic factors, a phenomenon in which exosomes may play a major role. Here, we investigated the potential of exosomes from human umbilical cord-derived MSCs (hUC-MSCs-Exos) at alleviating OA.

Methods

hUC-MSCs were isolated, cultured, and identified based on the expression of MSC markers and multipotency differentiation. hUC-MSCs-Exos were harvested from hUC-MSC conditioned medium using a sequential centrifugation method. Transmission electron microscopy, dynamic light scattering, flow cytometry, and western blotting were used to identify the exosomes. The effects of hUC-MSCs-Exos on the proliferation and migration of human chondrocytes were evaluated using the cell counting kit-8, EdU555 cell proliferation kit, and transwell assays. Annexin V-FITC/PI staining and flow cytometry were used to evaluate the effect of exosomes on chondrocyte apoptosis. An in vitro model of human articular chondrocytes treated with interleukin 1 beta (IL-1ß) was used to evaluate the effects of exosomes; analyses involved using quantitative real-time polymerase chain reaction (qRT-PCR), immunofluorescence, and western blotting. The role of exosomes in macrophage polarization was examined in the monocyte cell line, THP-1. Rats with surgically induced OA (ACLT+pMMx method) were intra-articularly injected with hUC-MSCs-Exos. The efficacy of exosome injections was assessed using hematoxylin and eosin and safranin-0 and fast green staining, and immunohistochemistry.

Results

We confirmed the superior efficacy of hUC-MSCs-Exos at promoting chondrocyte proliferation and migration and inhibiting chondrocyte apoptosis. Additionally, hUC-MSCs-Exos reversed IL-1 $\beta$-induced injury in vitro. hUC-MSCs-Exos could inhibit the secretion of pro-inflammatory factors, promote the expression of anti-inflammatory factors, and regulate the polarization of macrophages. hUC-MSCs-Exos attenuated the progression of $\mathrm{OA}$ and prevented severe damage to the knee articular cartilage in the rat OA model.

Conclusions

hUC-MSCs-Exos exerted immunomodulatory and therapeutic effects in a rat model of OA. These exosomes derived from hUC-MSCs can potentially serve as treatments for OA.

\section{Background}


Osteoarthritis (OA) is one of the most common chronic joint diseases worldwide, affecting approximately $10 \%$ of men and $18 \%$ of women over 60 years of age(1). This age-related degenerative joint disease is characterized by the progressive destruction of the articular cartilage and cartilage loss caused by the disequilibrium between anabolism and catabolism of chondrocytes and the extracellular matrix (ECM)(2). Current treatment modalities for cartilage injuries, such as microfracture, mosaicplasty, and autologous chondrocyte implantation, are limited by donor site morbidity and inferior fibrocartilage repair(3). Furthermore, the inherent limited healing potential of cartilage tissue introduces significant challenges in its self-repair and remodeling. Therefore, the present treatment modalities for OA are limited by their inability to prevent and slow the progression of the disease(4).

Mesenchymal stem cells (MSCs), with their remarkable potential for proliferation and differentiation into various cell lineages, are recognized as a promising cell source in the treatment of various degenerative, inflammatory, and autoimmune diseases(5). MSCs are primarily isolated from tissues such as the bone marrow, adipose tissue, and umbilical cord. These cells are widely used in cell-based therapy or in tissue engineering to treat cartilage lesions and $O A$, which have been extensively investigated in both animal and human studies(6-9). Additionally, hUC-MSCs are considered a better choice compared to MSCs owing to the painless collection procedure, absence of ethical issues with respect to their use, and high proliferation potential, cell vitality, and paracrine potential(9-11). However, due to drawbacks such as the safety issues of MSCs, inconvenient storage and transportation, and differentiation for non-therapeutic purposes, there is limited research on their applications. Similar to all cell-based therapies, MSC-based therapy is associated with significant operational costs and challenges as cell-based medicine requires stringently monitored manufacturing and storage to ensure optimal viability and vitality of the cells at all stages (harvest, expansion, storage, and delivery to patients) $(3,12,13)$. Moreover, many studies have shown that MSCs exert their therapeutic effects, i.e., reduction of cellular injury and enhanced repair through the secretion of reparative factors $(12,14,15)$. Co-culture studies further demonstrated that MSCs secrete trophic factors to promote chondrocyte proliferation and matrix synthesis (16).

Exosomes have been identified as the principal agents mediating the therapeutic efficacy of MSCs in several diseases $(14,17)$. Exosomes are extracellular vesicles with a size range of 30 to $200 \mathrm{~nm}$ (average $\sim 100 \mathrm{~nm}$ ) that have an endosomal origin. Many exosome components are derived from their cells of origin; these include DNA, RNA, lipids, metabolites, as well as cytosolic and cell-surface proteins(17). Repair of osteochondral defects using MSC-derived exosomes was characterized by increased proliferation and infiltration of chondrocytes, and enhanced ECM synthesis $(18,19)$. Wu et al. showed that exosomes derived from infrapatellar fat pad MSCs could protect the cartilage from damage and ameliorate gait abnormalities observed in OA mice(20). Wang et al confirmed that exosomes from embryonic MSCs exert a beneficial therapeutic effect on OA by balancing the synthesis and degradation of chondrocyte-derived ECM(21). Nevertheless, ameliorating inflammation constitutes an important component of strategies aimed at promoting cartilage repair $(18,22)$. Zhang et al. found that exosomes derived from MSCs have an immunomodulatory effect and can induce M2 macrophages to infiltrate the defects and synovium of $\mathrm{OA}$ cartilage, reduce the infiltration of $\mathrm{M} 1$ macrophages, and downregulate the expression of IL-1 $\beta$ and TNF- $\alpha$, thereby inhibiting the inflammatory response in OA. Similar 
immunoregulatory roles of exosomes derived from MSCs have been observed in inflammatory bowel disease(23), spinal cord injury(24), and diabetic peripheral neuropathy(25). The potential use of MSCderived exosomes as a novel cell-free therapy for the treatment of OA is worthy of recognition.

Therefore, we investigated the regenerative and immunomodulatory effects of hUC-MSCs-Exos in a rat model of OA.

\section{Methods}

The umbilical cords were obtained from mothers of healthy full-term fetuses who had provided written informed consent at The Second Hospital of Jilin University. Consent for publication was also obtained from all donors. This study was performed in accordance with the Guidelines for Stem Cell Research and Clinical Translation (2016, ISSCR) and approved by the Ethics Committee of The Second Hospital of Jilin University (approval 2019-142).

\section{Cell culture}

hUC-MSCs were isolated as described previously(10). Briefly, umbilical cords were washed with phosphate-buffered saline (PBS, Thermo Fisher Scientific, USA) containing 5\% penicillin/streptomycin solution (P/S, $100 \mathrm{IU} / \mathrm{mL}$ penicillin, $100 \mathrm{IU} / \mathrm{mL}$ streptomycin; Thermo Fisher Scientific). Umbilical cord tissues were carefully dissected to remove vessels and then cut into pieces of approximately $1 \mathrm{~mm}^{3}$, followed by suspension in Dulbecco's Modified Eagle medium containing nutrient mixture F-12 (DMEM/F12; Thermo Fisher Scientific) supplemented with $10 \%$ fetal bovine serum (FBS; Thermo Fisher Scientific), $1 \% \mathrm{P} / \mathrm{S}$, and $10 \mathrm{ng} / \mathrm{mL}$ basic fibroblast growth factor (bFGF; PeproTech, UK). The suspension was used to coat the pre-incubated petri dishes. The explant-seeded plates were incubated for $1-2 \mathrm{~h}$ in an inverted position to ensure the proper attachment of the explants. Thereafter, $3 \mathrm{~mL}$ of fresh medium was gently added to the seeded plate, followed by incubation at $37{ }^{\circ} \mathrm{C}$ in a humidified $5 \% \mathrm{CO}_{2}$ incubator. The medium was changed every three days. An outgrowth of adherent cells from the tissue explants were observed after 1 week of seeding. After 10-14 days, the tissue explants were removed from the petri dishes, leaving behind the attached cells. When the hUC-MSCs had proliferated to approximately $80 \%$ confluence, cell harvesting was carried out using $0.25 \%$ EDTA-Trypsin (Thermo Fisher Scientific), followed by centrifugation at $300 \times g$ for $5 \mathrm{~min}$. Harvested cells were further grown to passage 4 for all the experiments. An inverted fluorescence microscope (Olympus IX71, Japan) was used to monitor the phenotype and growth characteristics of the hUC-MSCs. These cells were sub-cultured and used as cell sources in subsequent experiments. Human chondrocytes (C-12710) for the knee and hip joint cartilage tissue were obtained from PromoCell's cell culture facility and cultured in a chondrocyte growth medium (PromoCell, Germany). At 24 hours after cell seeding, we changed the medium into that with $10 \mathrm{ng} / \mathrm{mL}$ interleukin 1 beta (IL-1 $\beta$ ) (PeproTech, USA) according to the experimental design. The nest day, the medium with or without $4 \times 10^{7}$ particles/mL hUC-MSCs-Exos was added according to the experimental design, and the cells were collected and analyzed after $48 \mathrm{~h}$. Normal cultured chondrocytes served as control. Human monocytic THP-1 cells (TIB-202, ATCC; USA) were cultured in RPMI-1640 medium 
(Thermo Fisher Scientific) supplemented with $10 \%$ FBS, and 1\% P/S. Based on a previous study(26), THP1 cells were induced into M0 macrophages by incubation with $25 \mathrm{ng} / \mathrm{mL}$ phorbol 12-myristate 13-acetate (PMA; Sigma-Aldrich, USA) for $24 \mathrm{~h}$. Once the cells became adherent, they were polarized to M1 macrophages by incubation with lipopolysaccharide (LPS; Sigma-Aldrich; $100 \mathrm{ng} / \mathrm{mL}$ ) for $24 \mathrm{~h}$. M0 and M1 macrophages $\left(5 \times 10^{5}\right.$ cells) were exposed to hUC-MSCs-Exos ( $4 \times 10^{7}$ particles) in RPMI-1640 medium.

\section{Multipotency assay of hUC-MSCs}

In order to assess the multi-lineage differentiation potential of the hUC-MSCs, a multipotency assay was performed. The differentiation potentials of hUC-MSCs were evaluated based on previous studies(10). For chondrogenic differentiation, hUC-MSCs spheres were generated using a hanging drop culture, utilizing $20 \mu \mathrm{L}$ of $8 \times 10^{6}$ cells $/ \mathrm{mL}$ solution; spheres were cultured in chondrogenic induction medium consisting of HG-DMEM, 10\% FBS, $6.25 \mu \mathrm{g} / \mathrm{mL}$ insulin, $10 \mathrm{ng} / \mathrm{mL}$ transforming growth factor-beta 1 (PeproTech), and $50 \mathrm{nM}$ of ascorbate-2-phosphate (Sigma-Aldrich). The culture medium was replaced every three days. Three weeks after chondrogenic induction, cartilage was detected with toluidine blue (Dingguo, China) staining according to the manufacturer's protocol. For adipogenic differentiation, the hUC-MSCs from passage 5 were grown in the adipogenic medium containing high glucose-Dulbecco's Modified Eagle Medium (HG-DMEM; Thermo Fisher Scientific), 10\% FBS, 1\% P/S, $1 \mu \mathrm{M}$ dexamethasone (SigmaAldrich), $0.5 \mathrm{mM}$ isobutyl-methylxanthine (Sigma-Aldrich), $10 \mu \mathrm{M}$ insulin (Sigma-Aldrich), and $200 \mu \mathrm{M}$ indomethacin (Sigma-Aldrich) for 2 weeks. For osteogenic differentiation, the hUC-MSCs from passage 5 were cultured in an osteogenic medium consisting of HG-DMEM, 10\% FBS, 1\% P/S, $0.1 \mu \mathrm{M}$ dexamethasone, $50 \mu \mathrm{M}$ ascorbate-2-phosphate (Sigma-Aldrich), and $10 \mathrm{nM} \beta$-glycerophosphate (SigmaAldrich). After 4 weeks of culture, Alizarin Red S (Sigma-Aldrich) staining was used to detect the mineralization of calcium salt accumulated in the hUC-MSCs, as described previously(27). Adipogenic differentiation in the hUC-MSCs was confirmed by the appearance of lipid inclusion vacuoles, which were stained with neutral Oil Red O (Sigma-Aldrich), as described previously(27). Images were collected by using an inverted fluorescence microscope. Images were collected by using an inverted fluorescence microscope.

\section{Immunofluorescence staining and flow cytometry assay}

Cells were incubated with mouse anti-human monoclonal antibodies: anti-CD31(1:100; MA1-26196, Thermo Fisher Scientific), anti-CD 45 (1:100; 16-0451-85, Thermo Fisher Scientific), anti-CD90 (1:100; PA582115, Thermo Fisher Scientific), anti-CD105(1:100; MA5-17041, Thermo Fisher Scientific), antiCD73(1:100; 41-0200, Thermo Fisher Scientific), and anti-COL2A1 (1:100; MA5-12781, Thermo Fisher Scientific); anti-CD44(1:1000; MAB7045, R\&D Systems, UK) and anti-MMP13 (1:1000; MAB511, R\&D Systems, UK); anti-ACAN (1:1000; NB-600-504, Novus Biologicals, UK); and rabbit anti-human monoclonal antibodies: anti-SOX9 (1:2000; AB5535, Sigma-Aldrich), anti-ADAMTS5 (1:1000; Novus Biologicals), and COL1A2 (1:1000; 95855S, Cell Signaling Technology, USA). Subsequently, cells were incubated with the corresponding goat anti-mouse secondary antibody or goat anti-rabbit secondary antibody (Alexa Fluor 
488 and 555; 1:1000; Cell Signaling Technology). Cells were then counterstained with Hoechst 33342 dye $(10 \mu \mathrm{g} / \mathrm{mL}$; Thermo Fisher Scientific) and visualized under an inverted fluorescence microscope. For surface marker detection, a flow cytometry assay was performed. Cells were incubated with primary mouse anti-human monoclonal antibodies (1:100): anti-CD31, anti-CD45, anti-CD90, anti-CD105, antiCD44, and anti-CD73. At the end of incubation, cells were washed twice with PBS and then incubated with goat anti-mouse secondary antibody (Alexa Fluor 488; 1:2000). After washing twice with PBS, target cells were analyzed on a FACS Calibur instrument (BD Biosciences, USA). Cells that were stained with only the secondary antibody served as a negative control.

\section{Isolation and identification of exosomes}

When hUC-MSCs reached $50-60 \%$ confluence, they were washed with PBS and cultured in conditioned medium containing DMEM/F-12, 10\% non-exosomes-FBS (SBI, USA), $1 \% \mathrm{P} / \mathrm{S}$, and $10 \mathrm{ng} / \mathrm{mL} \mathrm{bFGF}$ for an additional $48 \mathrm{~h}$ at $37{ }^{\circ} \mathrm{C}$ in a humidified $5 \% \mathrm{CO}_{2}$ incubator. Exosome isolation was carried out using ultracentrifugation(28). Collected culture suspension was transferred to conical tubes for centrifugation at $300 \times g$ for $10 \mathrm{~min}$ at $4{ }^{\circ} \mathrm{C}$ to obtain the pellet. The supernatant was centrifuged a second time at 2,000 $\times g$ for 10 mins at $4{ }^{\circ} \mathrm{C}$ to further remove cell debris, followed by a third time at $10,000 \times g$ for 30 min at 4 ${ }^{\circ} \mathrm{C}$ to further remove apoptotic bodies and other organelles. Finally, the supernatant was ultracentrifuged at $120,000 \times g$ for $90 \mathrm{~min}$ at $4{ }^{\circ} \mathrm{C}$ in a SW32Ti rotor (Beckman Coulter, USA) to obtain the exosome pellet. The pellet containing hUC-MSCs-Exos was resuspended with PBS and centrifuged at 120,000 $\times g$ for 90 min at $4{ }^{\circ} \mathrm{C}$. The supernatant was discarded, and the pellet containing hUC-MSCs-Exos was resuspended with $400 \mu \mathrm{L}$ PBS and stored at $-80^{\circ} \mathrm{C}$. The particle number of hUC-MSCs-Exos was quantified using an exosome ELISA complete kit (CD81 detection; System Biosciences, USA) following the manufacturer's instructions. Morphology of the exosomes was observed by using transmission electron microscopy (TEM). The size distribution of exosomes was measured using ZETASIZER Nano series-Nano-ZS (Malvern Instruments, UK), and was analyzed using Zetasizer software (Malvern Instrument). The exosomes were loaded onto copper grids and contrasted using $2 \%$ uranyl acetate (Sigma-Aldrich), dried, and observed using a TECNAI 12 TEM (FEl, USA). Antibodies against CD63 (1:1000; ab271286, Abcam, UK) and CD81 (1:1000; ab79559, Abcam) proteins were used to analyze the incorporation of each protein into exosomes using western flow cytometry and blotting. PKH26 (Sigma-Aldrich) was used to track the cellular entry of exosomes.

\section{Western blotting}

Cells or exosomes were lysed in a radioimmunoprecipitation assay (RIPA) and phenylmethylsulfonyl fluoride (PMSF) buffer (ratio: RIPA:PMSF = 100:1; Beyotime, China) for $30 \mathrm{~min}$ on ice and centrifuged at $13,000 \times g$ for 20 min at $4{ }^{\circ} \mathrm{C}$. The supernatant was collected, and the total protein concentration was determined using a BCA kit (Beyotime). An amount of $20 \mu \mathrm{g}$ total protein was fractionated by using $10 \%$ SDS-PAGE and the separated proteins were blotted onto $0.22-\mu \mathrm{m}$ polyvinylidene fluoride (PVDF) membranes (Beyotime). Membranes were then blocked with 5\% skim milk in TBST (10 mM Tri-HCL, 150 $\mathrm{mM} \mathrm{NaCl}, 0.25 \%$ Tween-20, $\mathrm{pH} 7.5$ ) at room temperature for $1 \mathrm{~h}$, followed by overnight incubation with 
the following primary antibodies: anti-COL2A1, anti-SOX9, anti-ACAN, anti-MMP13, anti-COL1A2, and antiADAMTS5; anti-GAPDH (1:1000, Cell Signaling Technology) served as the protein-loading control. After washing with TBST, the membranes were incubated for $1 \mathrm{~h}$ at room temperature with the secondary antimouse or anti-rabbit IgG antibody (1:2000, Cell Signaling Technology). After three washes with TBST, signals were detected by chemiluminescence using the ECL-Plus detection system (TransGen, China). The relative amount of proteins on the blots was determined using ImageJ (National Institutes of Health, USA).

\section{qRT-PCR}

Total RNA was extracted from cells using Trizol (Thermo Fisher Scientific) according to the manufacturer's instructions and reverse-transcribed into cDNA using a reverse transcription kit (Roche, Switzerland), following the manufacturer's instructions. qRT-PCR was performed with FastStart Universal SYBR Green Master (ROX; Roche) on a Real-Time PCR System (CFX Connect Real-Time System, BIO-RAD, Germany). The fold-changes in cDNA levels of the target gene, after normalization to the levels of the reference gene $G A P D H$, were determined using the $2^{-\triangle \triangle C T}$ method(29). qRT-PCR primers for COL2A1 (NM_033150.2), SOX9 (NM_000346.3), ACAN(NM_013227.2), MMP13 (NM_002427.3), ADAMTS5

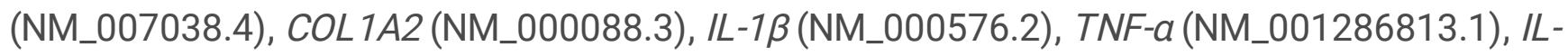
10 (NM_000572.2), ARG1 (NM_000045.2), and GAPDH(NM_001256799.2) were purchased from Guangzhou GeneCopoeia (China).

\section{Chondrocyte proliferation, migration, and apoptosis assays}

The effect of hUC-MSCs-Exos on chondrocyte proliferation was evaluated using the cell counting kit-8 (CCK-8; Dojindo, Japan), as described previously(30). Cell proliferation curves were constructed by measuring the amount of formazan dye generated by the activity of cellular dehydrogenase using a microplate reader (TECAN, Austria) at a wavelength of $450 \mathrm{~nm}$. The incorporated EdU was detected through a reaction between the alkyne group of EdU and the fluorescent azide in a copper-catalyzed azide-alkyne cycloaddition. Chondrocytes treated with $4 \times 10^{7}$ particles/mL hUC-MSCs-Exos were measured using an EdU-555 cell proliferation kit (Ribobio, China) following the manufacturer's instructions and visualized under an inverted fluorescence microscope. EdU-free chondrocytes served as a negative control. The effect of chondrocyte stimulation with $4 \times 10^{7}$ particles $/ \mathrm{mL}$ hUC-MSCs-Exos was evaluated using a transwell assay. In brief, after digestion and counting, approximately $5 \times 10^{4}$ cells were seeded into the upper chamber of a 24-well 8- $\mu \mathrm{m}$-pore-size transwell plate (Corning, USA). Subsequently, $600 \mu \mathrm{L}$ of DMEM/F-12 medium containing exosomes was added into the lower chamber and $400 \mu \mathrm{L}$ of DMEM/F-12 medium was added into the upper chamber before incubation for $24 \mathrm{~h}$ at $37^{\circ} \mathrm{C}$. The upper chamber was then fixed with $4 \%$ PFA for $15 \mathrm{~min}$, stained with $0.5 \%$ crystal violet for $10 \mathrm{~min}$, and washed with PBS three times. The upper surface of the upper chamber was carefully wiped using a cotton swab to remove cells that had not migrated to the lower surface. Images were collected by using an inverted fluorescence microscope. The chondrocytes stained with crystal violet that migrated to the lower chamber were counted by using ImageJ software. Chondrocyte apoptosis was detected using a FITC- 
annexin $\triangle$ apoptosis detection kit (BD Biosciences) according to the manufacturer's instructions. After 24 h of passage 3 chondrocyte seeding, the DMEM/F-12 medium was replaced with medium containing 10 $\mathrm{ng} / \mathrm{mL}$ IL-1 $\beta$. The nest day, the medium with or without $4 \times 10^{7}$ particles $/ \mathrm{mL}$ hUC-MSCs-Exos was added according to the experimental design, and the cells were collected and analyzed after $48 \mathrm{~h}$. Normal cultured chondrocytes served as control. Chondrocyte apoptosis was detected by following the manufacturer's instructions. Samples were analyzed on a FACS Calibur instrument.

\section{Animal studies}

Male Sprague-Dawley rats (approximately 12 weeks old) weighing $300-350 \mathrm{~g}$, housed in a specific pathogen-free (SPF) animal laboratory with $12: 12 \mathrm{~h}$ light/dark cycle, controlled temperature environment $\left(20-26^{\circ} \mathrm{C}\right)$, and steady humidity $(40-70 \%)$, were used. All protocols involving animals were performed in accordance with the ethical guidelines of the National Institutes of Health Guide for the Care and Use of Laboratory Animals and approved by the ethics committee of the Guangdong Medical Laboratory Animal Center Care and Use Committee (B202007-15). Based on a previous report, OA in our rat model was surgically induced by using the anterior cruciate ligament transection in combination with medial meniscectomy (ACLT+pMMx) method, without damaging the tibial surface(31). All rats were randomly divided into three groups: (1) Normal (without surgery; received articular cavity injection of $100 \mu \mathrm{L}$ PBS on day 1 and 4 of every week from the 5th to 8th week; 6 knee joints from 3 rats, $n=6$ ); (2) OA (rats underwent surgery and received articular cavity injection of $100 \mu \mathrm{L}$ PBS on day 1 and 4 of every week from the 5th to 8th week after surgery; 6 knee joints from 3 rats, $n=6$ ); (3) OA+ Exos (rats received articular cavity injection of $100 \mu \mathrm{L}$ hUC-MSCs-Exos in PBS, $10^{11}$ particles $/ \mathrm{mL}$ of exosomes on day 1 and 4 of every week from the 5 th to 8 th week after surgery; 6 knee joints from 3 rats, $n=6$ ). Eight weeks after surgery, rats were sacrificed, and the knee samples were harvested for the evaluation of disease progression.

\section{Histology and immunohistochemical analysis}

The tibiofemoral joints were removed, and the femoral condyles were fixed in neutral-buffered formalin (containing $4 \%$ formaldehyde) for $24 \mathrm{~h}$ after the knee samples were harvested. The fixed femoral condyles were decalcified in EDTA (Leagene, China) for 28 days (refreshed every day) before dehydration using graded ethanol (Beijing Chemical Works, China), vitrification using dimethyl benzene (Beijing Chemical Works), paraffin embedding, and tissue sectioning (5 $\mu \mathrm{m})$, prior to staining with H\&E for morphological evaluation. Tissue sections were stained using a safranin- 0 and fast green staining kit (Solarbio, China) and examined for matrix proteoglycan and overall joint morphology. Immunohistochemistry was performed to evaluate the function of articular chondrocytes. Rabbit antiCOL2A1, rabbit anti-MMP13, mouse anti-CD86, and rabbit anti-CD163 were used as primary antibodies (1:200; Abcam). After washing off excess primary antibodies, samples were incubated with secondary antibodies conjugated with HRP: HRP-labeled goat anti-mouse IgG (1:200; Beyotime) or goat anti-rabbit IgG antibody (1:200; Beyotime). Peroxidase-conjugated antibody (1:600; Beyotime) was diluted in 1\% $(\mathrm{w} / \mathrm{v})$ BSA solution and incubated for $1 \mathrm{~h}$. A DAB detection system (Beyotime) was used to visualize the 
sections. For quantification analysis, positively stained cells were counted in three randomly selected fields by using ImageJ software and the mean number of cells per high power field (cells/HPF) was determined. Based on safranin- 0 and fast green staining, the osteoarthritis research society international (OARSI) score, which is a well-recognized histological scoring system(31), was used by three independent pathologists in a blinded manner to evaluate the OA progression in every sample of each group.

\section{Statistical analyses}

All experiments were performed with at least three biological replicates. Data are expressed as the mean \pm standard deviation. Statistical analyses were performed using GraphPad Prism 7.0 software. Multiple comparisons were analyzed using analysis of variance (ANOVA) or Student's $t$ test followed by Bonferroni correction. $\mathrm{P}<0.05$ was considered statistically significant.

\section{Results}

\section{Isolation and identification of hUC-MSCs}

Cells migrated out from the UC pieces between days 7 and 14 and proliferated in the tissue culture plates (Fig. 1a). Isolated cells showed the formation of colonies with fibroblast morphology. Cells at passage 4 were identified and used in subsequent experiments. After reaching $80-90 \%$ confluence, cells adopted a spindle-like shape (Fig. 1b). The cells were collected, pooled, and subjected to phenotypic characterization and multipotency assays. When cultured in chondrogenic, osteogenic, or adipogenic medium, cells could be induced to differentiate along the chondrogenic, osteogenic, or adipogenic pathways, respectively. Chondrogenic potential was confirmed by sectioning beads and staining sulfated glycosaminoglycans using toluidine blue (Fig. 1C). Osteogenic potential was confirmed using calcium mineral deposits stained with Alizarin red (Fig. 1d). Adipogenic potential was evaluated by the observation of small cytoplasmic lipid droplets stained using Oil Red O (Fig. 1e). Following the criteria for identifying MSCs, we analyzed the surface markers of the cells. Analysis of surface antigen expression using immunofluorescence staining demonstrated that the cells were positive for CD44, CD73, CD90, and CD105, and negative for CD31 and CD45 (Fig. 1f). Flow cytometry confirmed the expression rate of the cell differentiation markers CD31 (2.3\%), CD44 (95.2\%), CD45 (3.2\%), CD73 (96.7\%), CD90 (94.3\%), and CD105 (98.2\%) (Fig. 1 g). The spindle-shaped cells that expressed the MSC differentiation markers CD44, CD73, CD90, and CD105 and possessed multipotent differentiation potentials towards chondrocytes, osteoblasts, and adipocytes were classified as hUC-MSCs.

\section{Isolation and identification of hUC-MSCs-Exos}

TEM revealed that the vesicles from hUC-MSCs exhibited a round-shaped morphology with a diameter of 30-200 nm (Fig. 2a). Nanoparticle tracking analysis revealed that $82.5 \%$ of vesicles displayed a particle size distribution between 20 and $200 \mathrm{~nm}$ (Fig. 2b). Flow cytometry confirmed the expression of the vesicle differentiation markers CD63 (78.1\%) and CD81 (79\%) (Fig. 2c, d). Western blotting also detected 
the presence of exosome marker proteins, namely CD63 and CD81 (Fig. 2e). All these data indicated that hUC-MSCs-Exos were successfully isolated.

\section{Exosomes promote chondrocyte proliferation, migration, and inhibit apoptosis}

To confirm whether the chondrocytes could internalize hUC-MSCs-Exos, hUC-MSCs-Exos were labeled using red fluorescent PKH26. Chondrocytes were then incubated for $24 \mathrm{~h}$ with $\mathrm{PKH} 26$-labeled exosomes. After washing with PBS, the PKH26-labeled exosomes were observed in the cytoplasm of the chondrocytes, confirming the internalization by chondrocytes. Almost all chondrocytes were positive for exosome internalization (Fig. 3a). After quantifying the exosomes using an exosome ELISA complete kit, proliferation was evaluated following the stimulation of chondrocytes with $0,0.5,1,2$, or $4 \times$ $10^{7}$ particles/mL of hUC-MSCs-Exos. Based on our concentration range, CCK-8 assay results confirmed that all concentrations of exosomes could promote the proliferation of chondrocytes in a dose-dependent manner (Fig. 3b). EdU assay results further showed that the proliferative ability of chondrocytes was notably enhanced by hUC-MSCs-Exos (Fig. 3c). The results of the transwell assay showed that the migratory ability of chondrocytes was markedly strengthened by hUC-MSCs-Exo treatment (Fig. 3d). Inhibition of apoptosis is an important factor in promoting cell proliferation. We used annexin VFITC/PI staining and flow cytometry to detect the effect of exosomes on the inhibition of chondrocyte apoptosis (Fig. 3e). IL-1 $\beta$ was used to induce apoptosis (22.24\%) and hUC-MSCs-Exo treatment decreased the apoptotic rate of chondrocytes (8.02\%). These results clearly demonstrated the superior efficacy of hUC-MSCs-Exos in promoting chondrocyte proliferation and matrix synthesis and inhibiting chondrocyte apoptosis.

\section{Exosomes reverse IL-1 $\beta$-induced chondrocyte injury}

We used a model of OA-like chondrocytes reported in a previous study(32). In our experiment, qPCR results showed that IL-1 $\beta$ could reduce the gene expression levels of COL2A1, SOX9, and ACAN and increase those of MMP13, ADAMTS5, and COL 1A2. The OA-like chondrocyte model was successfully constructed. Interestingly, the supplementation of hUC-MSCs-Exos in chondrocytes dramatically reversed the effect of IL-1 $\beta$ on the gene expression of COL2A1, SOX9, ACAN, MMP13, ADAMTS5, and COL1A2 (Fig. 4a), consistent with the results obtained from the immunofluorescence staining assay (Fig. 4b). Western blotting further confirmed that IL-1 $\beta$ could reduce protein expression of COL2A1, SOX9, and ACAN in chondrocytes and increase the expression of MMP13, ADAMTS5, and COL1A2 at the protein level. However, more importantly, results from western blotting confirmed that hUC-MSCs-Exos could reverse the effect of IL-1 $\beta$ on the COL2A1, SOX9, ACAN, MMP13, ADAMTS5, and COL1A2 proteins (Fig. 4c, d). In conclusion, exosomes could reverse the injury of chondrocytes induced by IL-1 $\beta$ in a model of OA-like chondrocytes in vitro.

\section{Role of exosomes in macrophage polarization}

In order to evaluate the role of exosomes in macrophage activity, we performed an in vitro assay using a polarization protocol of the THP-1 monocyte cell line. THP1 was induced into M0 and M1 macrophages 
using PMA and LPS. When the M0 and M1 macrophages were treated using hUC-MSCs-Exos, qPCR results showed that the expression levels of anti-inflammatory factors IL-10 and ARG1 increased, whereas the expression levels of IL-1 $\beta$ and TNF- $\alpha$ decreased significantly (Fig. 5a). Immunofluorescence showed that compared to the control group, the number of CD163-positive cells was significantly increased, and the number of CD86-positive cells was significantly decreased in M0 and M1 macrophages treated with exosomes (Fig. 5b).

\section{Exosomes attenuate OA progression}

In the rat model, OA was surgically induced using the ACLT+pMMx method. Rats underwent surgery and received articular cavity injection of PBS or hUC-MSCs-Exos from the 5th to 8th week after surgery (days 1 and 4 of every week). Eight weeks after the surgery, knee samples were harvested for the evaluation of disease progression (Fig. 6a). No adverse events occurred in any of the experimental groups. We verified the potential of hUC-MSCs-Exos for OA prevention in an OA rat model (Fig. 6b). In the OA+PBS group, H\&E and safranin- $O$ and fast green staining showed that the cartilage showed moderate surface irregularity, superficial fibrillation, loss of proteoglycan, and loss of cartilage in the superficial zone, but none of these effects were observed when the cartilage was treated with exosomes. Based on safranin-0 and fast green staining, the OARSI score was used to evaluate OA progression. The OARSI score of group OA+Exos was $1.12 \pm 0.26$ and that of group OA+PBS was $2.92 \pm 0.42$ (Fig. 6c). In the OA+PBS group, expression of COL2A1 in the cartilage decreased, whereas that of MMP13 was observed on the cartilage surface. In the OA+Exos group, joint wear and cartilage matrix loss were scarcely observed. Expression of COL2A1 and MMP13 in the cartilage was similar to that in the normal group, based on the cartilage surface (Fig. $6 \mathbf{b}, \mathbf{d}$, e). As indicated by CD86-positive cells, M1 macrophage numbers reduced in the cartilage of the OA+Exos group compared with that in the OA+PBS group (Fig. 6b, f). In contrast to the M1 macrophages, we observed an abundance of M2 macrophages, as indicated by CD163-positive cells in the cartilage of the OA+Exos group compared with those in the OA+PBS group (Fig. $6 \mathbf{b}, \mathbf{g}$ ). These data indicated that the exosomes attenuated OA progression and prevented severe damage to the knee articular cartilage in the rat OA model, which was caused by instability of the knee joint.

\section{Discussion}

$\mathrm{OA}$ is a prevalent chronic joint disease(1). Current therapies attempt to relieve the symptoms, but they cannot block or reverse the ongoing cartilage degeneration(2). Ideal treatment aiming toward achieving optimal OA joint repair should promote the regenerative properties of chondrocytes and eliminate the destructive effects of inflammation(33). Here, we report, for the first time, the effect of exosomes derived from hUC-MSCs on the treatment of OA. Our data demonstrated that hUC-MSCs-Exos slowed the progression of $\mathrm{OA}$ and prevented severe damage to knee articular cartilage in the rat OA model. We also demonstrated that hUC-MSCs-Exos balance the synthesis and degradation of the cartilage extracellular matrix and regulate macrophages. In the treatment of OA, exosomes derived from hUC-MSC offer many advantages. First, many previous studies have validated the safety of hUC-MSCs-Exos, which is crucial for the design of future clinical trials(34). We did not observe any adverse events in our OA model rats 
administered hUC-MSCs-Exos. Second, hUC-MSCs-Exos have been reported to play a role in repairing tissue damage in vital organs, especially in the kidney(35), liver(36), lung(37), and heart(38, 39). Our study demonstrated that direct administration of hUC-MSCs-Exos may effectively treat cutaneous wounding(28). Third, hUC-MSCs have been presented as the best source of exosomes because of the absence of ethical concerns, easy availability, non-invasive isolation with high yield, and greater differentiation and immunomodulatory potential $(10,38)$. Fourth, the high abundance of miRNAs in hUCMSCs-Exos plays an important role in maintaining cartilage homeostasis, promoting chondrogenesis, and regulating inflammation; these miRNAs include miRNA-21-5p $(40,41), 146 a-3 p(42$, 43), 26a-5p(44), 100-5p(20,45), and let-7a(46). The high-throughput sequencing results of exosomes in our study showed that the abovementioned miRNAs were abundant (results not shown). We obtained the exosomes of umbilical cord-derived MSCs from three volunteers by using an ultracentrifugation isolation method. The results of TEM, flow cytometry, and particle size analysis showed that the exosomes of hUCMSC were consistent with those reported in the literature $(17,28)$. We used the method of ultracentrifugation, with varying speed and time, to gradually remove impurities in the sample and achieve the separation and enrichment of exosomes, which is convenient for up-scaling and minimizes residues and impurities.

Previous studies have demonstrated that exosomes derived from the human bone marrow(47), adipose $(48)$ and embryonic $(21,49)$ tissue-derived MSCs, and amniotic fluid stem cells(50) could cause effective cartilage repair by inducing the migration and proliferation of chondrocytes and promoting cartilage matrix synthesis. Our results also showed that hUC-MSCs-Exos could promote the proliferation and migration of chondrocytes. At the lowest concentration of hUC-MSCs-Exos $\left(0.5 \times 10^{7}\right.$ particles $\left./ \mathrm{mL}\right)$, the effect of promoting the proliferation of chondrocytes was still observed, which was lower than the concentration of exosomes from other stem cells reported in the literature(30). Tao et al. found that exosomes derived from human synovial MSCs clearly promoted chondrocyte proliferation and migration. However, there was one crucial shortcoming: the inhibition of the synthesis of ECM proteins, including ACAN and collagen II(51). Exosomes derived from hUC-MSCs can modulate chondrocytes treated with IL$1 \beta$ to maintain the expression of chondrogenic markers (COL2A1, SOX9, and ACAN) and decrease MMP13, ADAMTS5, and COL1A2 expression. Therefore, hUC-MSCs-Exos can not only promote chondrocyte proliferation and matrix synthesis and inhibit chondrocyte apoptosis in vitro but also reverse IL-1 $\beta$-induced chondrocyte damage by balancing the synthesis and degradation of cartilage ECM.

Unilateral surgical ACLT+pMMx in mature rats leads to the development of progressive cartilage degeneration. For all rat models of surgically induced $O A$, it is recommended that skeletally mature animals, 12 weeks of age or older, be used to best mimic the development of OA in humans. This model is also sufficiently sensitive for the investigation of new therapeutic strategies that could prevent $O A$ progression(31). In this model, exosomes by themselves were demonstrated to be able to prevent lesion progression and exert a similar regenerative effect as exosomes from other stem cells(31). In our animal experiment, both hind limb knee joints of rats were surgically intervened for the construction of an $\mathrm{OA}$ model. Compared to other studies using animal models $(49,51)$, we increased the number of exosome 
injections to twice a week for four weeks. Results showed that increasing the frequency of exosome injection was effective to enhance the therapeutic effect of exosomes on OA arthritis.

$\mathrm{OA}$ is considered a multifactorial cartilage lesion disease within a chronic inflammatory microenvironment(18). Macrophage-associated inflammation is a driver of OA structural damage and progression. Hikmat et al. suggested that targeting macrophages and macrophage-associated inflammatory pathways may be an effective means to treat $\mathrm{OA}(52)$. Anti-inflammatory intervention to ameliorate inflammation has been shown to promote chondrocyte survival and mitigate the risk of development to post-traumatic OA. For example, it was reported that M2 macrophage polarization in $\mathrm{OA}$ synovium tissues supports the survival of chondrocytes by producing anti-inflammatory IL-10 to suppress adverse inflammation, whereas M1-polarized macrophages inhibit MSC chondrogenic differentiation via IL-6 in vitro(53). Our in vitro results also showed that hUC-MSCs-Exos can inhibit the

secretion of pro-inflammatory factors, promote the expression of anti-inflammatory factors, and regulate the polarization of macrophages. Additionally, MSC exosomes induced higher infiltration of CD163 ${ }^{+}$ regenerative $\mathrm{M} 2$ macrophages than that of inflammatory $\mathrm{CD}^{2} 6^{+} \mathrm{M} 1$ macrophages during articular cartilage repair.

Nevertheless, the effective dosage of exosomes needs to be optimized. To date, the active ingredients in exosomes are unknown. Further studies focusing on the efficacy of exosomes derived from a larger cohort of donors should be conducted. The observation time for animal model studies needs to be extended with an increased number of observation time points, to provide a more accurate reference for the application of exosomes in OA treatment. Since exosomes are complex carriers that contain DNA, RNA, lipids, metabolites, and various proteins, they exhibit therapeutic functions through immunomodulation, bioenergetics, or biochemical effects in various disease models. Hence, the mechanisms underlying their therapeutic effects in OA need to be investigated further.

\section{Conclusions}

In this study, we confirmed the efficacy of exosomes derived from hUC-MSCs at treating OA. Exosomes derived from hUC-MSCs can not only promote the proliferation and migration of chondrocytes in vitro but also reverse IL-1 $\beta$-associated damage. hUC-MSCs-Exos can inhibit the secretion of pro-inflammatory factors, promote the expression of anti-inflammatory factors, and regulate the polarization of macrophages. Exosomes derived from hUC-MSCs slowed the progression of early OA in vivo and prevented severe damage to the knee articular cartilage that arises as the result of the instability of the knee joint in the rat OA model (Fig. 7).

\section{Abbreviations}

Osteoarthritis (OA); extracellular matrix (ECM); mesenchymal stem cells (MSCs); phosphate-buffered saline (PBS); Dulbecco's Modified Eagle medium containing nutrient mixture F-12 (DMEM/F-12); fetal bovine serum (FBS); phorbol 12-myristate 13-acetate (PMA); lipopolysaccharide (LPS); transmission 
electron microscopy (TEM); radioimmunoprecipitation assay (RIPA); phenylmethylsulfonyl fluoride (PMSF); polyvinylidene fluoride (PVDF); cell counting kit-8 (CCK-8); specific pathogen-free (SPF); osteoarthritis research society international (OARSI); anterior cruciate ligament transection in combination with medial meniscectomy (ACLT+pMMx); analysis of variance (ANOVA)

\section{Declarations}

\section{Ethics declarations}

All donors of umbilical cords provided written informed consent prior to specimen collection and all relevant protocols were approved by the Ethics Committee of the Second Hospital of Jilin University (approval number 2019-142).

All experimental procedures on Sprague-Dawley rats were performed in accordance with the ethics committee of the Guangdong Medical Laboratory Animal Center Care and Use Committee (B202007-15), in compliance with the National Institutes of Health Guide for the Care and Use of Laboratory Animals.

\section{Consent for publication}

All donors of umbilical cords provided their consent for publication.

\section{Availability of data and materials}

The datasets used and/or analyzed in the current study are available from the corresponding author on reasonable request.

\section{Competing interests}

The authors declare that they have no competing interests.

\section{Funding}

This work was supported by the China Natural National Science Foundation (81970547, 81571199), and the Science and Technology Department of Jilin Province (20190101006JH).

\section{Authors' contributions}

GC, QL and YL conceived and designed the experiments. PL performed the experiments. PL, VL, and WJ wrote the manuscript. LS, and BL analyzed the data and prepared all the figures. ZX, GZ, LW, JZ, HW, QP, and ZL provided technical support. All authors reviewed and agreed upon the final manuscript.

\section{Acknowledgements}


Not applicable.

\section{References}

1. Hunter DJ, Bierma-Zeinstra S. Osteoarthritis. Lancet. 2019;393:1745-59.

2. Fellows CR, Matta C, Mobasheri A. Applying proteomics to study crosstalk at the cartilagesubchondral bone interface in osteoarthritis: Current status and future directions. EBioMedicine. 2016;11:2-4.

3. Jiang YZ, Zhang SF, Qi YY, Wang LL, Ouyang HW. Cell transplantation for articular cartilage defects: principles of past, present, and future practice. Cell Transplant. 2011;20:593-607.

4. Roseti L, Desando G, Cavallo C, Petretta M, Grigolo B. Articular cartilage regeneration in osteoarthritis. Cells. 2019;8:1305.

5. Pittenger MF, Mackay AM, Beck SC, Jaiswal RK, Douglas R, Mosca JD, et al. Multilineage potential of adult human mesenchymal stem cells. Science. 1999;284:143-7.

6. Jiang Y, Jahagirdar BN, Reinhardt RL, Schwartz RE, Keene CD, Ortiz-Gonzalez XR, et al. Pluripotency of mesenchymal stem cells derived from adult marrow. Nature. 2002;418:41-9.

7. Jo CH, Lee YG, Shin WH, Kim H, Chai JW, Jeong EC, et al. Intra-articular injection of mesenchymal stem cells for the treatment of osteoarthritis of the knee: a proof-of-concept clinical trial. Stem Cells. 2014;32:1254-66.

8. Kurth TB, Dell'accio F, Crouch V, Augello A, Sharpe PT, De Bari C. Functional mesenchymal stem cell niches in adult mouse knee joint synovium in vivo. Arthritis Rheum. 2011;63:1289-300.

9. Matas J, Orrego M, Amenabar D, Infante C, Tapia-Limonchi R, Cadiz MI, et al. Umbilical cordderived mesenchymal stromal cells (MSCs) for knee osteoarthritis: Repeated MSC dosing is superior to a single MSC dose and to hyaluronic acid in a controlled randomized phase I/II trial. Stem Cells Transl Med. 2019;8:215-24.

10. Zhao G, Liu F, Lan S, Li P, Wang L, Kou J, et al. Large-scale expansion of Wharton's jelly-derived mesenchymal stem cells on gelatin microbeads, with retention of self-renewal and multipotency characteristics and the capacity for enhancing skin wound healing. Stem Cell Res Ther. 2015;6:38.

11. $\mathrm{Xu} \mathrm{J}$, Sun $M$, Tan $Y$, Wang $H$, Wang $H$, Li P, et al. Effect of matrix stiffness on the proliferation and differentiation of umbilical cord mesenchymal stem cells. Differentiation. 2017;96:30-9.

12. Phinney DG, Pittenger MF. Concise review: MSC-derived exosomes for cell-free therapy. Stem Cells. 2017;35:851-8. 
13. Herberts CA, Kwa MS, Hermsen HP. Risk factors in the development of stem cell therapy. J Transl Med. 2011;9:29.

14. Lai RC, Yeo RWY, Lim SK. Mesenchymal stem cell exosomes. Semin Cell Dev Biol. 2015;40:82-8.

15. Caplan Al, Dennis JE. Mesenchymal stem cells as trophic mediators. J Cell Biochem. 2006;98:1076-84.

16. Wu L, Leijten JC, Georgi N, Post JN, van Blitterswijk CA, Karperien M. Trophic effects of mesenchymal stem cells increase chondrocyte proliferation and matrix formation. Tissue Eng Part A. 2011;17:1425-36.

17. Kalluri R, LeBleu VS. The biology, function, and biomedical applications of exosomes. Science. 2020;367:eaau6977.

18. Zhang S, Chuah SJ, Lai RC, Hui JHP, Lim SK, Toh WS. MSC exosomes mediate cartilage repair by enhancing proliferation, attenuating apoptosis and modulating immune reactivity. Biomaterials. 2018;156:16-27.

19. Mianehsaz E, Mirzaei HR, Mahjoubin-Tehran M, Rezaee A, Sahebnasagh R, Pourhanifeh MH, et al. Mesenchymal stem cell-derived exosomes: a new therapeutic approach to osteoarthritis? Stem Cell Res Ther. 2019;10:340.

20. Wu J, Kuang L, Chen C, Yang J, Zeng WN, Li T, et al. miR-100-5p-abundant exosomes derived from infrapatellar fat pad MSCs protect articular cartilage and ameliorate gait abnormalities via inhibition of mTOR in osteoarthritis. Biomaterials. 2019;206:87-100.

21. Wang Y, Yu D, Liu Z, Zhou F, Dai J, Wu B, et al. Exosomes from embryonic mesenchymal stem cells alleviate osteoarthritis through balancing synthesis and degradation of cartilage extracellular matrix. Stem Cell Res Ther. 2017;8:189.

22. Zhao X, Zhao Y, Sun X, Xing Y, Wang X, Yang Q. Immunomodulation of MSCs and MSC-derived extracellular vesicles in osteoarthritis. Front Bioeng Biotechnol. 2020;8:575057.

23. Schena F, Gambini C, Gregorio A, Mosconi M, Reverberi D, Gattorno M, et al. Interferon-y-dependent inhibition of B cell activation by bone marrow-derived mesenchymal stem cells in a murine model of systemic lupus erythematosus. Arthritis Rheum. 2010;62:2776-86.

24. Lankford KL, Arroyo EJ, Nazimek K, Bryniarski K, Askenase PW, Kocsis JD. Intravenously delivered mesenchymal stem cell-derived exosomes target M2-type macrophages in the injured spinal cord. PLoS One. 2018;13:e0190358.

25. Fan B, Li C, Szalad A, Wang L, Pan W, Zhang R, et al. Mesenchymal stromal cell-derived exosomes ameliorate peripheral neuropathy in a mouse model of diabetes. Diabetologia. 2020;63:431-43. 
26. Genin M, Clement F, Fattaccioli A, Raes M, Michiels C. M1 and M2 macrophages derived from THP1 cells differentially modulate the response of cancer cells to etoposide. BMC Cancer. 2015;15:577.

27. Li P, Liu F, Wu C, Jiang W, Zhao G, Liu L, et al. Feasibility of human hair follicle-derived mesenchymal stem cells/CultiSpher(®)-G constructs in regenerative medicine. Cell Tissue Res. 2015;362:69-86.

28. Zhao G, Liu F, Liu Z, Zuo K, Wang B, Zhang Y, et al. MSC-derived exosomes attenuate cell death through suppressing AIF nucleus translocation and enhance cutaneous wound healing. Stem Cell Res Ther. 2020;11:174.

29. Livak KJ, Schmittgen TD. Analysis of relative gene expression data using real-time quantitative PCR and the 2(-Delta C(T)) method. Methods. 2001;25:402-8.

30. Zhu Y, Wang Y, Zhao B, Niu X, Hu B, Li Q, et al. Comparison of exosomes secreted by induced pluripotent stem cell-derived mesenchymal stem cells and synovial membrane-derived mesenchymal stem cells for the treatment of osteoarthritis. Stem Cell Res Ther. 2017;8:64.

31. Gerwin N, Bendele AM, Glasson S, Carlson CS. The OARSI histopathology initiative recommendations for histological assessments of osteoarthritis in the rat. Osteoarthritis Cartilage. 2010;18 Suppl 3:S24-34.

32. Meng F, Zhang Z, Chen W, Huang G, He A, Hou C, et al. MicroRNA-320 regulates matrix metalloproteinase-13 expression in chondrogenesis and interleukin-1 $\beta$-induced chondrocyte responses. Osteoarthritis Cartilage. 2016;24:932-41.

33. Vonk LA, van Dooremalen SFJ, Liv N, Klumperman J, Coffer PJ, Saris DBF, et al. Mesenchymal stromal/stem cell-derived extracellular vesicles promote human cartilage regeneration. Theranostics. 2018;8:906-20.

34. Sun $L, X u$ R, Sun X, Duan $Y$, Han $Y$, Zhao $Y$, et al. Safety evaluation of exosomes derived from human umbilical cord mesenchymal stromal cell. Cytotherapy. 2016;18:413-22.

35. Zhang R, Yin L, Zhang B, Shi H, Sun Y, Ji C, et al. Resveratrol improves human umbilical cordderived mesenchymal stem cells repair for cisplatin-induced acute kidney injury. Cell Death Dis. 2018;9:965.

36. Shao M, Xu Q, Wu Z, Chen Y, Shu Y, Cao X, et al. Exosomes derived from human umbilical cord mesenchymal stem cells ameliorate IL-6-induced acute liver injury through miR-455-3p. Stem Cell Res Ther. 2020;11:37.

37. Zheng Y, Liu J, Chen P, Lin L, Luo Y, Ma X, et al. Exosomal miR-22-3p from human umbilical cord blood-derived mesenchymal stem cells protects against lipopolysaccharid-induced acute lung injury. Life Sci. 2021;269:119004. 
38. Yin S, Ji C, Wu P, Jin C, Qian H. Human umbilical cord mesenchymal stem cells and exosomes: bioactive ways of tissue injury repair. Am J Transl Res. 2019;11:1230-40.

39. Ma J, Zhao Y, Sun L, Sun X, Zhao X, Sun X, et al. Exosomes derived from Akt-modified human umbilical cord mesenchymal stem cells improve cardiac regeneration and promote angiogenesis via activating platelet-derived growth factor D. Stem Cells Transl Med. 2017;6:51-9.

40. Zhu H, Yan X, Zhang M, Ji F, Wang S. miR-21-5p protects IL-1 $\beta$-induced human chondrocytes from degradation. J Orthop Surg Res. 2019;14:118.

41. Hu SL, Chang AC, Huang CC, Tsai CH, Lin CC, Tang CH. Myostatin promotes interleukin-1 $\beta$ expression in rheumatoid arthritis synovial fibroblasts through inhibition of miR-21-5p. Front Immunol. 2017;8:1747.

42. Guan YJ, Li J, Yang X, Du S, Ding J, Gao Y, et al. Evidence that miR-146a attenuates aging- and trauma-induced osteoarthritis by inhibiting Notch1, IL-6, and IL-1 mediated catabolism. Aging Cell. 2018;17:e12752.

43. Al-Modawi RN, Brinchmann JE, Karlsen TA. Multi-pathway Protective effects of MicroRNAs on human chondrocytes in an in vitro model of osteoarthritis. Mol Ther Nucleic Acids. 2019;17:776-90.

44. Rasheed Z, Al-Shobaili HA, Rasheed N, Mahmood A, Khan MI. MicroRNA-26a-5p regulates the expression of inducible nitric oxide synthase via activation of NF-KB pathway in human osteoarthritis chondrocytes. Arch Biochem Biophys. 2016;594:61-7.

45. Luo P, Jiang C, Ji P, Wang M, Xu J. Exosomes of stem cells from human exfoliated deciduous teeth as an anti-inflammatory agent in temporomandibular joint chondrocytes via miR-100-5p/mTOR. Stem Cell Res Ther. 2019;10:216.

46. Sun F, Yang Q, Weng W, Zhang Y, Yu Y, Hong A, et al. Chd4 and associated proteins function as corepressors of Sox9 expression during BMP-2-induced chondrogenesis. J Bone Miner Res. 2013;28:1950-61.

47. Mao G, Zhang Z, Hu S, Zhang Z, Chang Z, Huang Z, et al. Exosomes derived from miR-92a-3poverexpressing human mesenchymal stem cells enhance chondrogenesis and suppress cartilage degradation via targeting WNT5A. Stem Cell Res Ther. 2018;9:247.

48. Tofiño-Vian M, Guillén MI, Pérez Del Caz MD, Silvestre A, Alcaraz MJ. Microvesicles from human adipose tissue-derived mesenchymal stem cells as a new protective strategy in osteoarthritic chondrocytes. Cell Physiol Biochem. 2018;47:11-25.

49. Zhang S, Chu WC, Lai RC, Lim SK, Hui JH, Toh WS. Exosomes derived from human embryonic mesenchymal stem cells promote osteochondral regeneration. Osteoarthritis Cartilage. 2016;24:2135-40. 
50. Zavatti M, Beretti F, Casciaro F, Bertucci E, Maraldi T. Comparison of the therapeutic effect of amniotic fluid stem cells and their exosomes on monoiodoacetate-induced animal model of osteoarthritis. Biofactors. 2020;46:106-17.

51. Tao SC, Yuan T, Zhang YL, Yin WJ, Guo SC, Zhang CQ. Exosomes derived from miR-140-5poverexpressing human synovial mesenchymal stem cells enhance cartilage tissue regeneration and prevent osteoarthritis of the knee in a rat model. Theranostics. 2017;7:180-95.

52. Daghestani HN, Pieper CF, Kraus VB. Soluble macrophage biomarkers indicate inflammatory phenotypes in patients with knee osteoarthritis. Arthritis Rheumatol. 2015;67:956-65.

53. Fahy N, de Vries-van Melle ML, Lehmann J, Wei W, Grotenhuis N, Farrell E, et al. Human osteoarthritic synovium impacts chondrogenic differentiation of mesenchymal stem cells via macrophage polarisation state. Osteoarthritis Cartilage. 2014;22:1167-75.

\section{Figures}




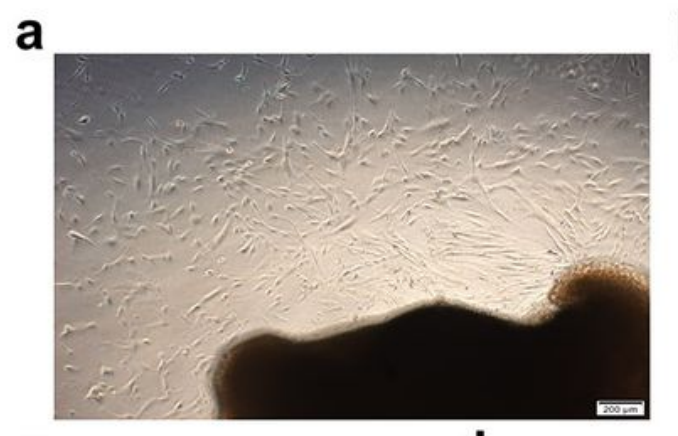

C

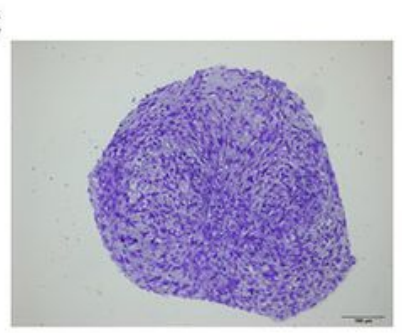

d
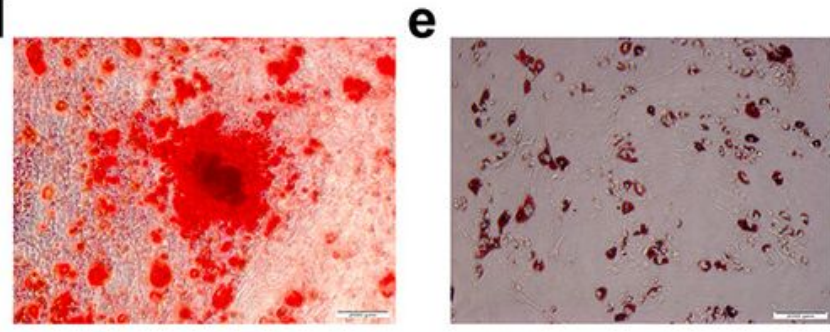

f
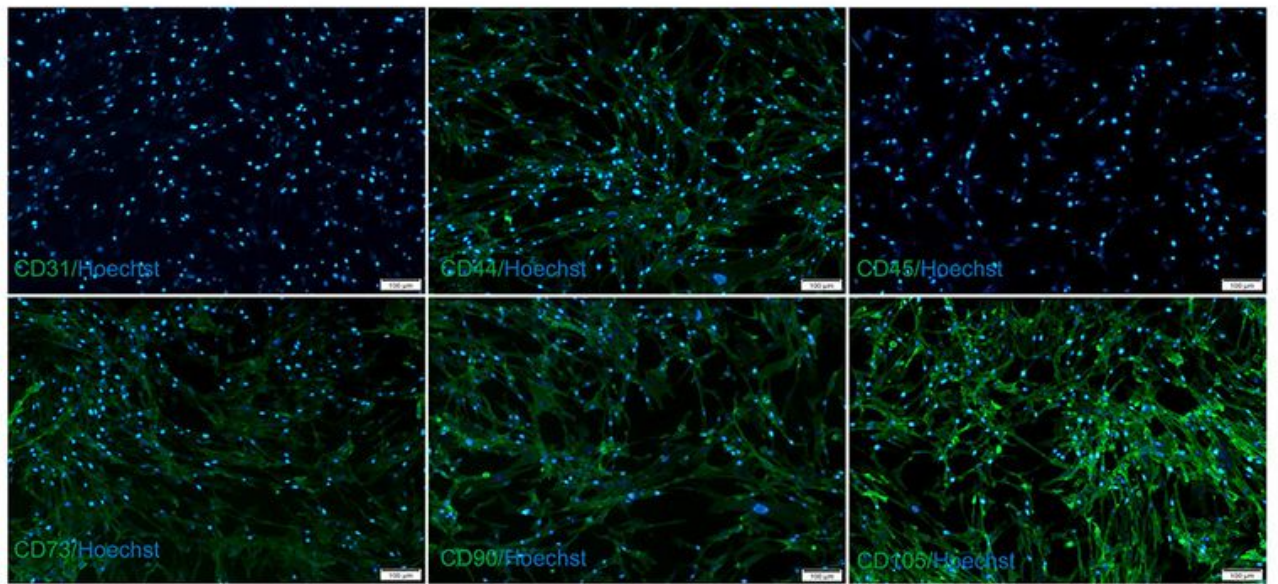

g
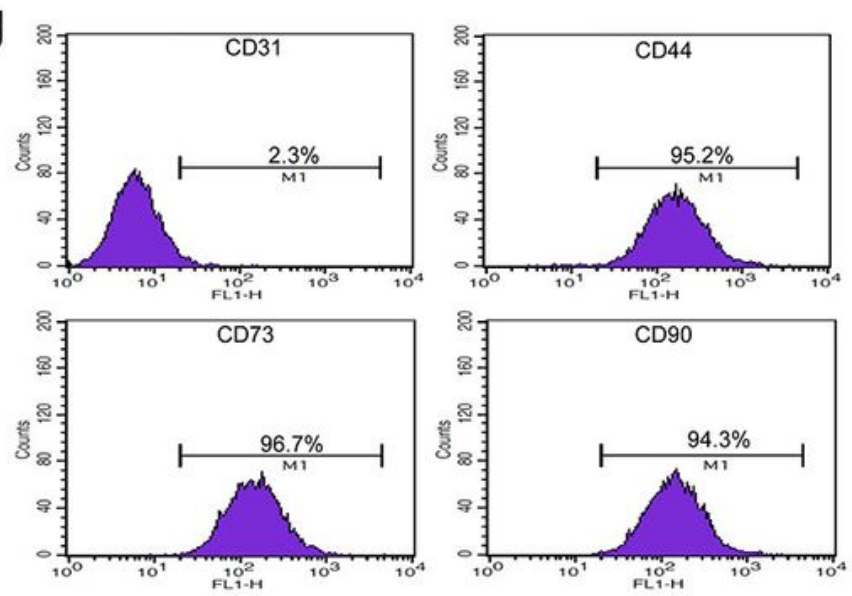
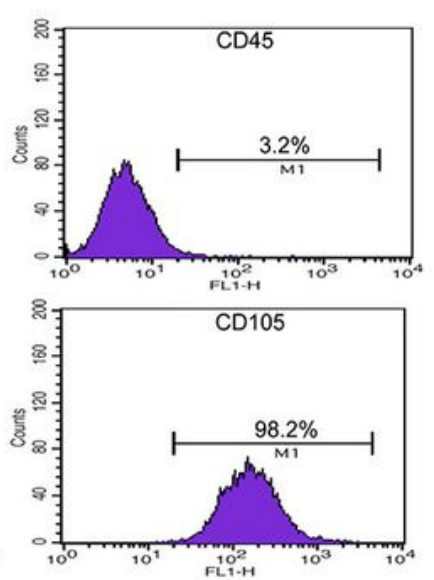

\section{Figure 1}

Characterization of human umbilical cord-derived mesenchymal stem cells (hUC-MSCs). a hUC-MSCs migrated out from the UC pieces on day 12, and exhibited spindle shape. scale bar: $200 \mu \mathrm{m}$. b At passage 5, hUC-MSCs also exhibited spindle shape morphology. Scale bar: $50 \mu \mathrm{m}$. c The hUC-MSCs exhibiting multipotent differentiation capacity for chondrogenesis. scale bar: $100 \mu \mathrm{m}$. d The hUC-MSCs exhibiting multipotent differentiation capacity for osteogenesis. scale bar: $200 \mu \mathrm{m}$. e The hUC-MSCs exhibiting 
multipotent differentiation capacity for adipogenesis. scale bar: $200 \mu \mathrm{m}$. f Staining of hUC-MSC surface markers on the spindle-shaped cells. The spindle-shaped cells expressed CD44, CD73, CD90, and CD105, and do not express CD34 and CD45. Hoechst 33342 staining was used for detecting the localization of cell nuclei (blue-color). Scale bar: $100 \mu \mathrm{m}$. e Flow cytometry assay of the hUC-MSCs revealed the expression of the hUC-MSC differentiation markers, CD34 (2.3\%), CD44 (95.2\%), CD45 (3.2\%), CD73 (96.7\%), CD90 (94.3\%), and CD105 (98.2\%).

\section{a}

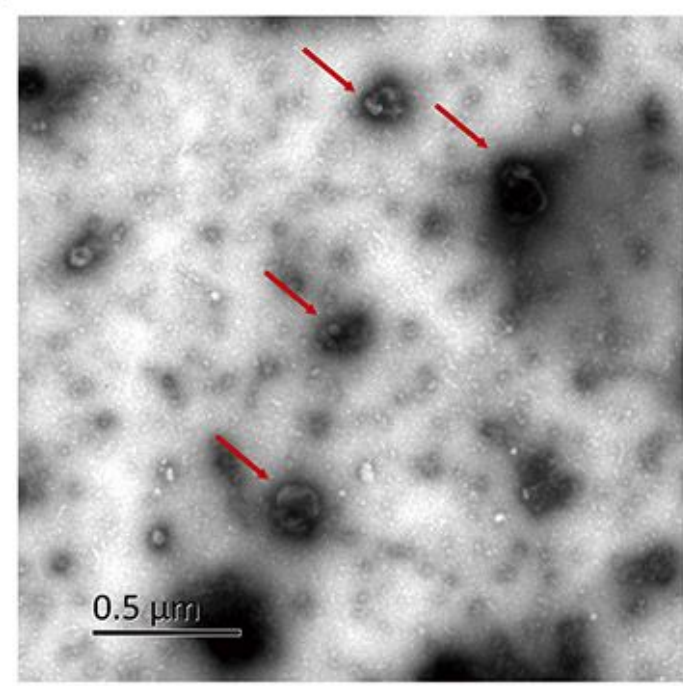

C

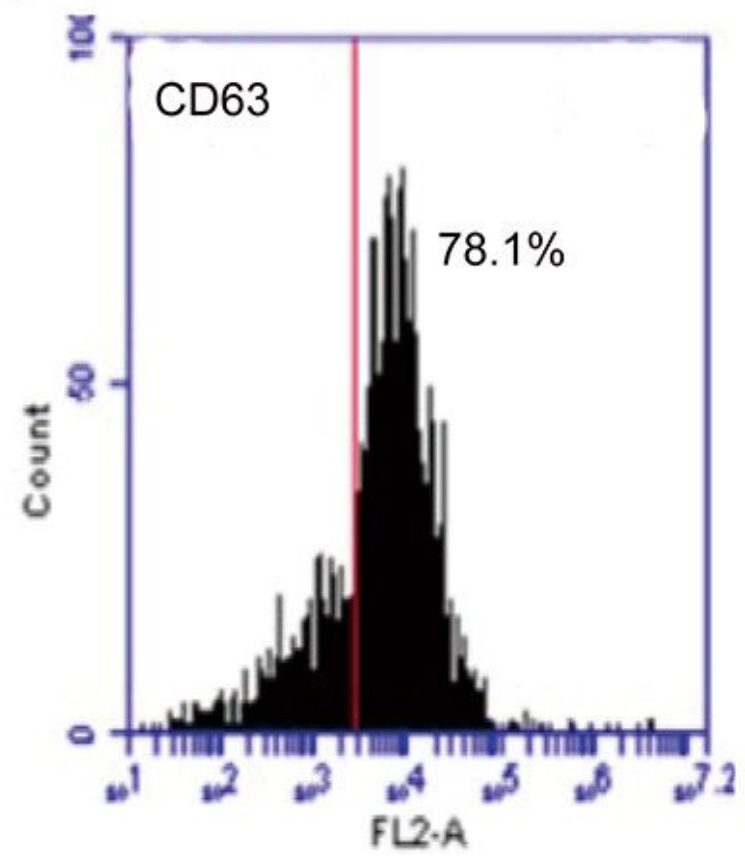

b

Size Distribution by Intensity

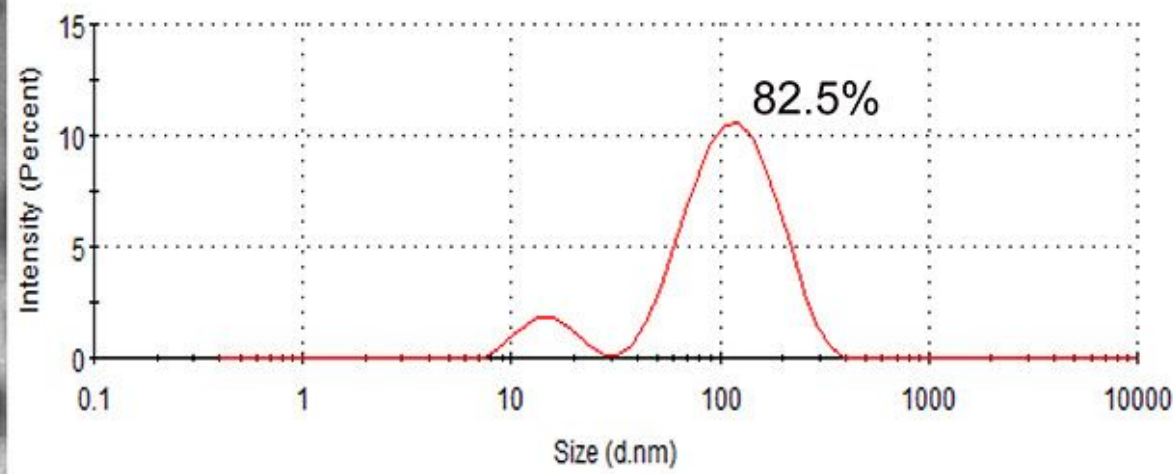

d

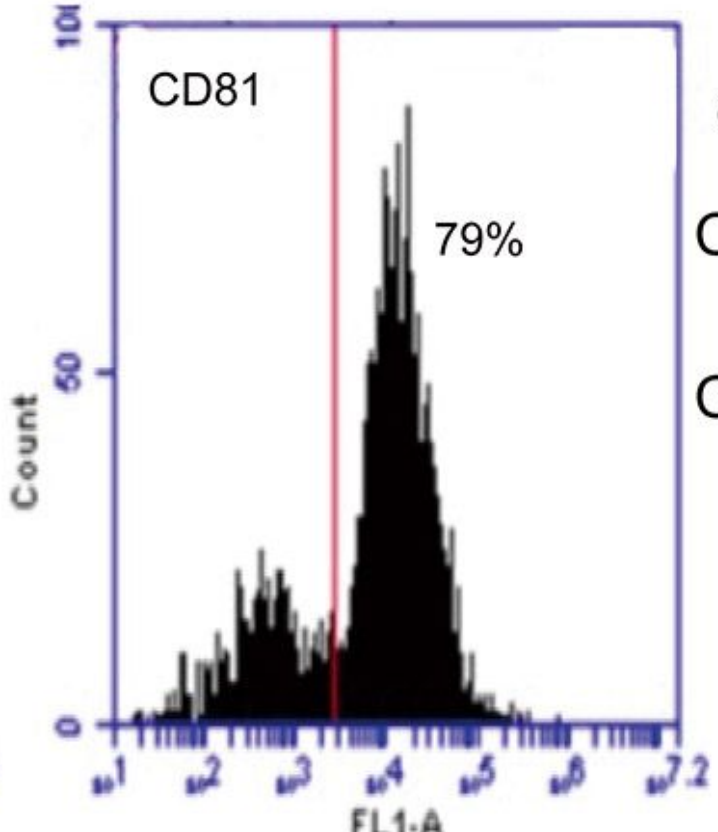

$\mathbf{e}$

CD63

CD81

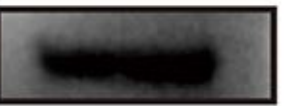

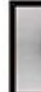

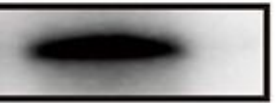

Figure 2

Characterization of exosomes derived from hUC-MSCs (hUC-MSCs-Exos). a Morphology of exosomes observed by transmission electron microscopy (TEM). Scale bar: $0.5 \mu \mathrm{m}$. b Particle size distribution of exosomes measured by dynamic light scattering (DLS). c This experiment was repeated independently 
three times and representative results are shown. $d$ Flow cytometry assay of the hUC-MSCs-Exos revealed expression rate of the hUC-MSCs-Exos differentiation markers, CD63 (78.1\%), CD81 (79\%). e Western blot indicated positive CD63, CD81 protein expression in hUC-MSCs-Exos.

a

\section{C}
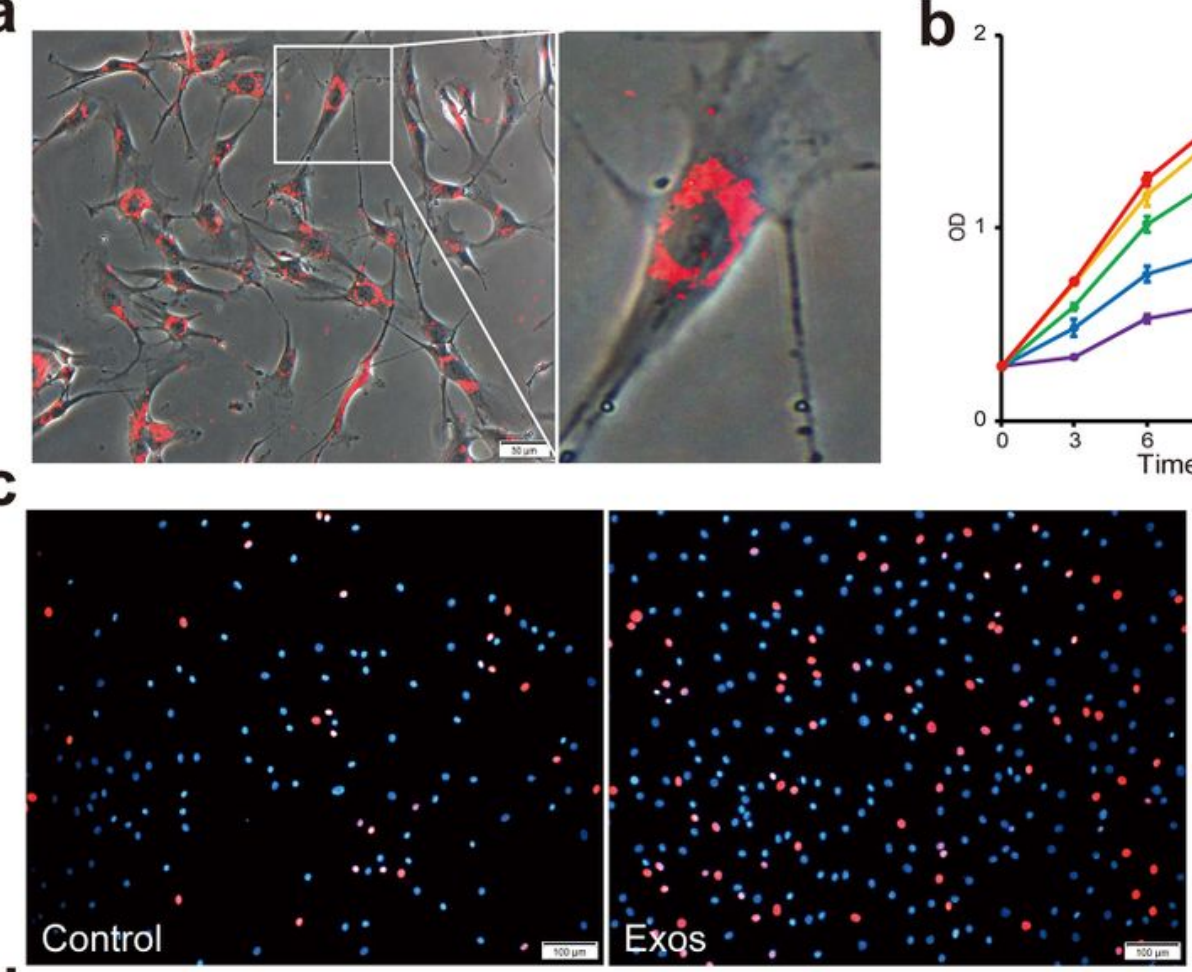

d

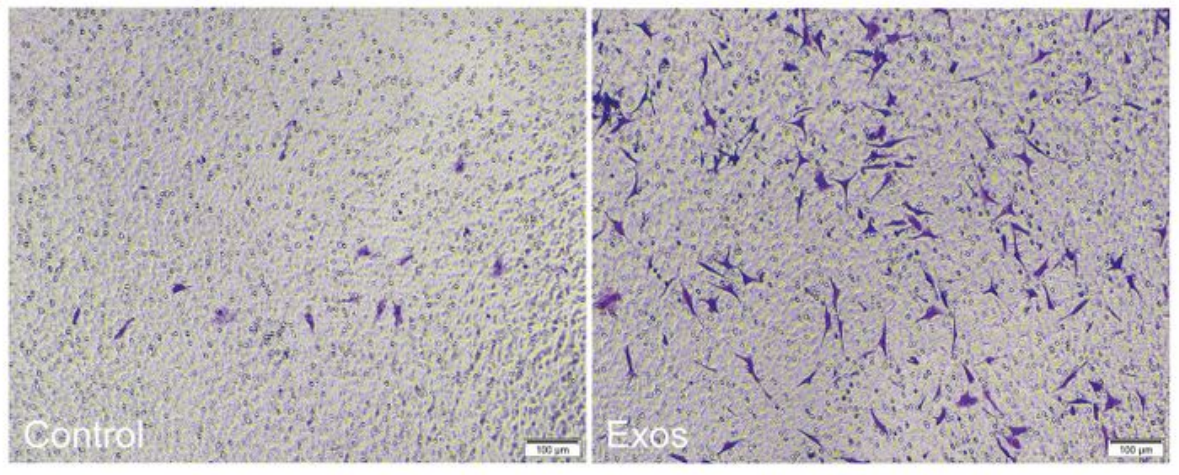

e
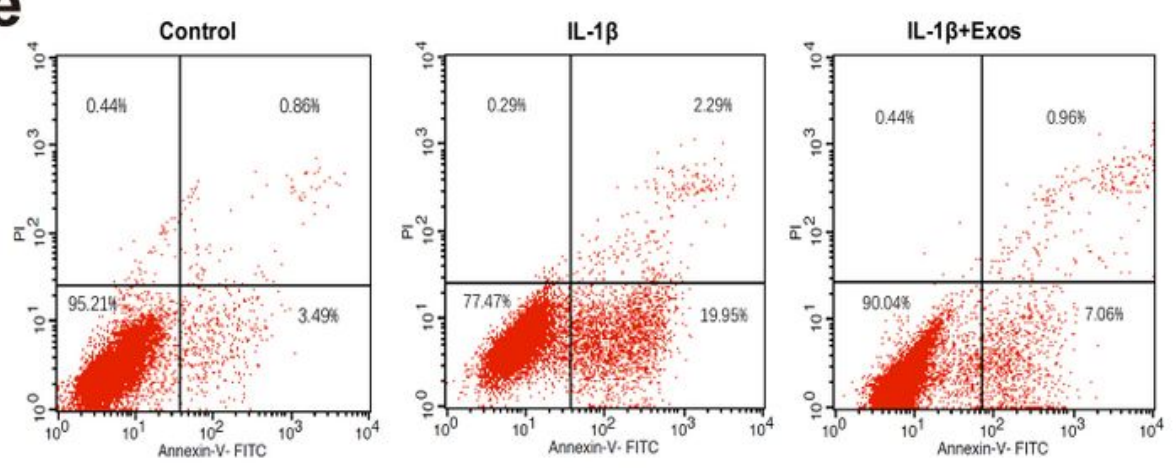

b

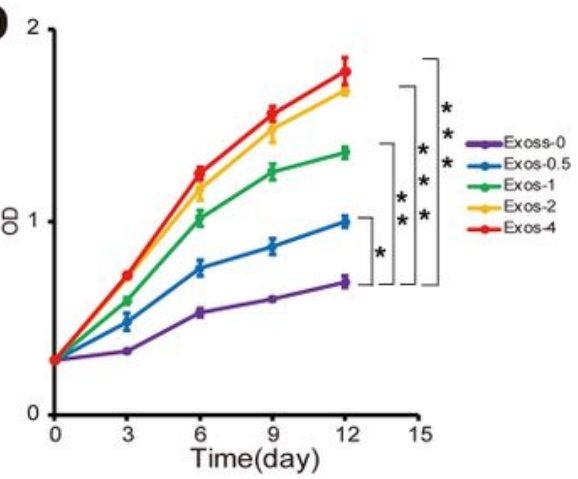

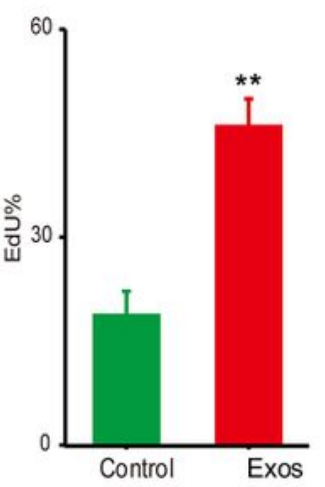
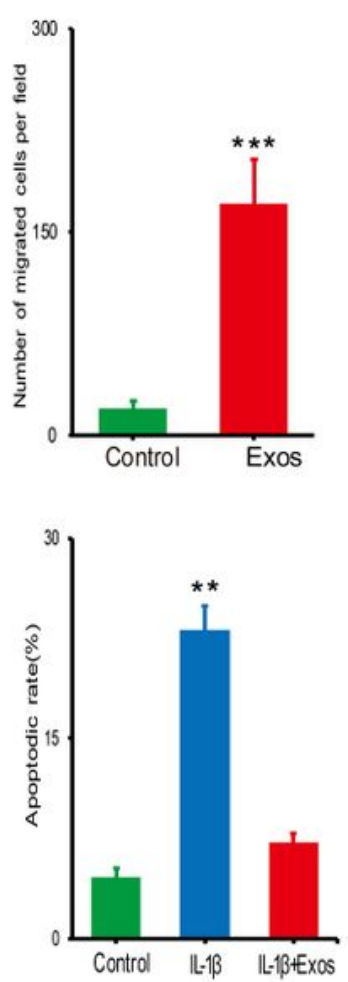

Figure 3

Effects of hUC-MSCs-Exos on proliferation and migration in chondrocytes. a Representative immunofluorescence photomicrograph of PKH26 (red)-labeled exosomes absorbed by chondrocytes 
(light field) on the right is an enlarged image of chondrocytes with exosomes internalized. Scale bar: 50 $\mu \mathrm{m}$. b hUC-MSCs-Exos were treated with chondrocytes at different concentrations. Quantitative data are presented as means \pm SD of three independent experiments. ${ }^{\star} P<0.05$, ${ }^{\star *} \mathrm{P}<0.01$, ${ }^{\star \star *} \mathrm{P}<0.001$. c EdUpositive cells and representative images of fields showing red-colored proliferative cells. Hoechst 33342 staining was performed to detect nuclear localization (blue color). Scale bar: $100 \mu \mathrm{m}$. Percentage of EdU was plotted as the mean $\pm S D$ of triplicate samples from 3 independent experiments. ${ }^{*} P<0.01$. $d$ Migrated chondrocytes were stained by crystal violet. Scale bar: $100 \mu \mathrm{m}$. Migrated chondrocytes was plotted as the mean \pm SD of triplicate samples from 3 independent experiments. ${ }^{* \star *} P<0.001$. e Annexin V-PI staining combined with flow cytometry assay to detect anti-apoptosis of hUC-MSCs-Exos in IL-1 $\beta$ treated chondrocytes. ${ }^{* *} \mathrm{P}<0.01$. 


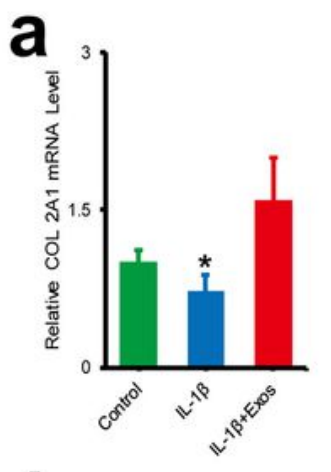

b

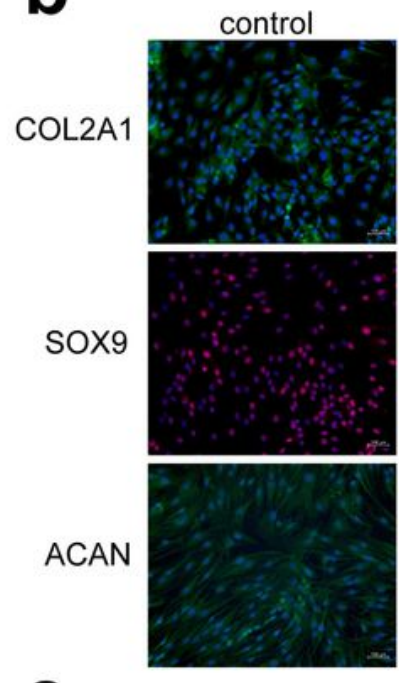

C

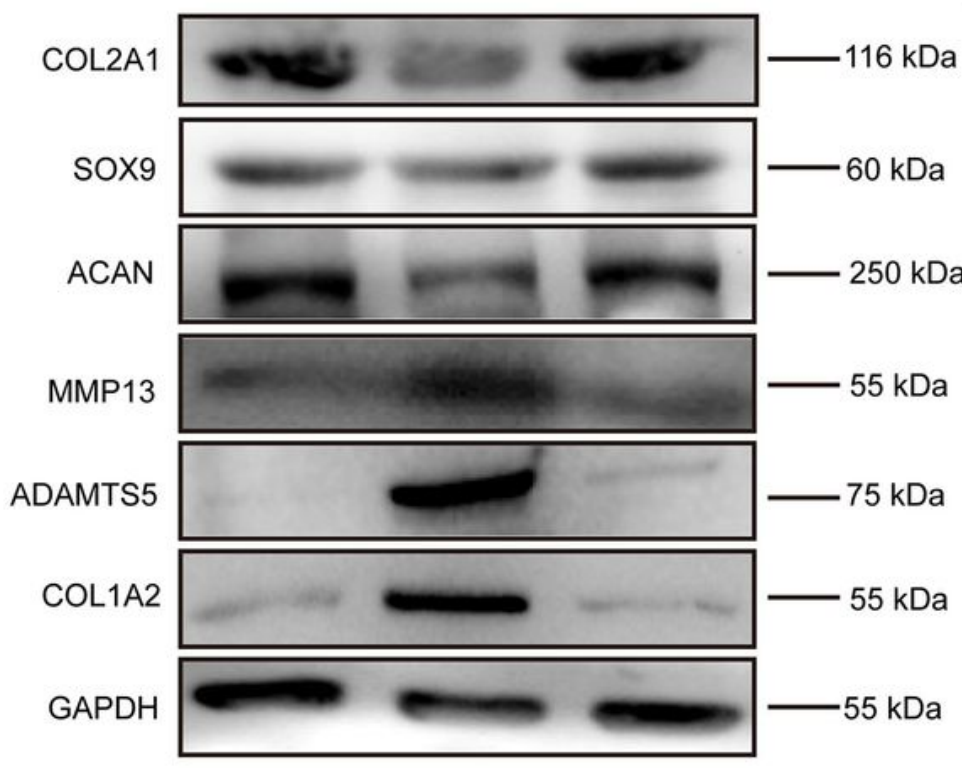

$\mathrm{IL}-1 \beta$

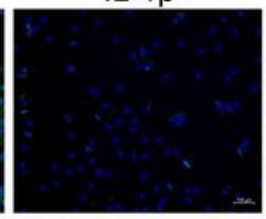

$\mathrm{IL}-1 \beta+$ Exos

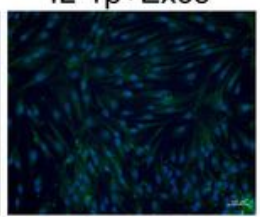

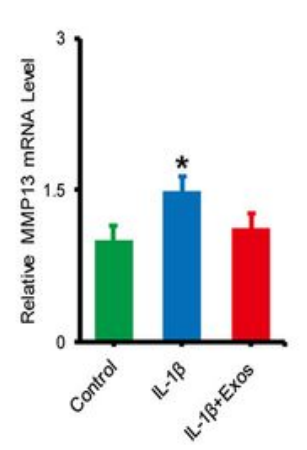
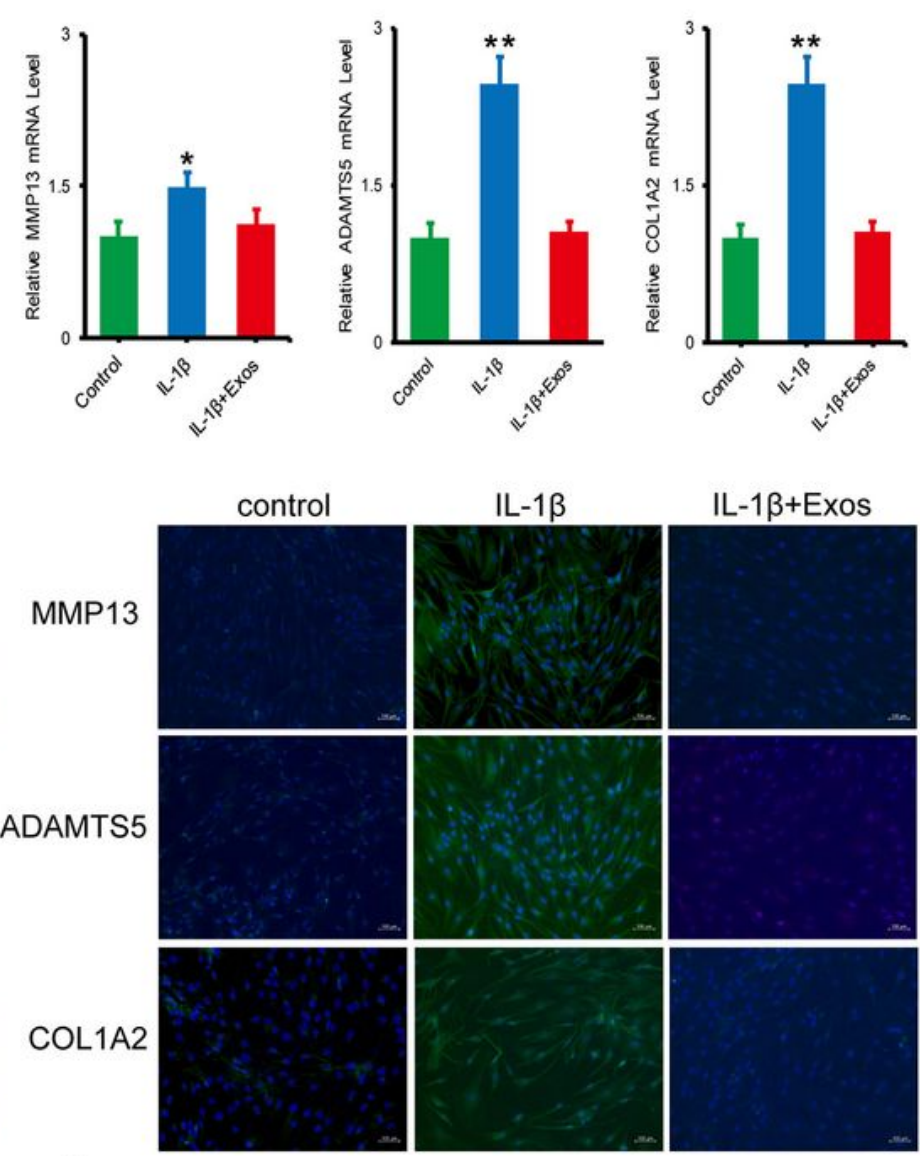

IL-1 $\beta$

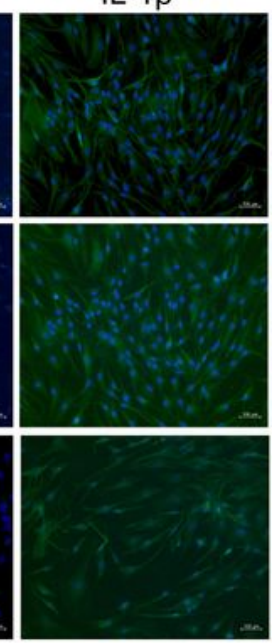

$\mathrm{IL}-1 \beta+$ Exos
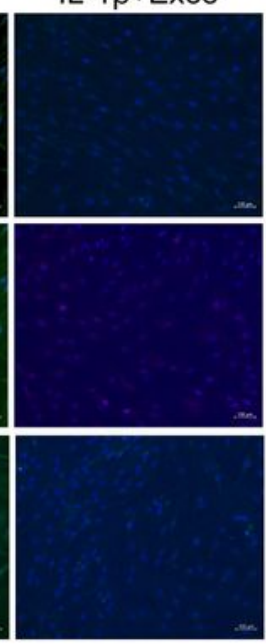

d
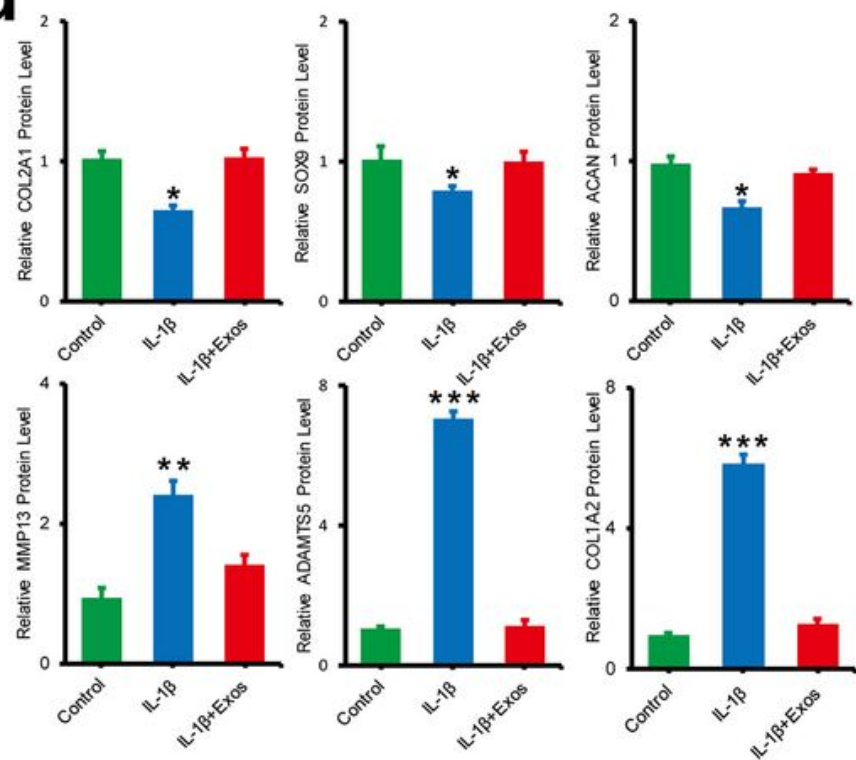

\section{Figure 4}

Exosomes reverse IL-1 $\beta$-induced chondrocyte injury. a Gene expression changes in chondrocytes of COL2A1, SOX9, ACAN, MMP-13, ADAMTS5, and COL1A2 after stimulation with IL-1 $\beta$ and exosomes. This experiment was repeated three times. ${ }^{*} P<0.05$, ${ }^{\star \star} P<0.01$ compared to untreated chondrocytes. $b$ COL2A1-, ACAN-, MMP-13-, ADAMTS5-, and COL1A2-positive cells and representative images of fields showing green-colored cytoplasm of chondrocytes in each group. SOX9-positive cells and representative 
images of fields showing red-colored nucleus of chondrocytes in each group. Hoechst 33342 staining was performed to detect nuclear localization (blue color). Scale bar: $100 \mu \mathrm{m}$. c, d Protein expression levels of COL2A1, ACAN, MMP-13, ADAMTS5, and COL1A2 in chondrocytes were detected using western blotting. This experiment was repeated three times. ${ }^{*} P<0.05,{ }^{\star} P P<0.01,{ }^{*} * \mathrm{P}<0.001$ compared to untreated chondrocytes.

\section{a}
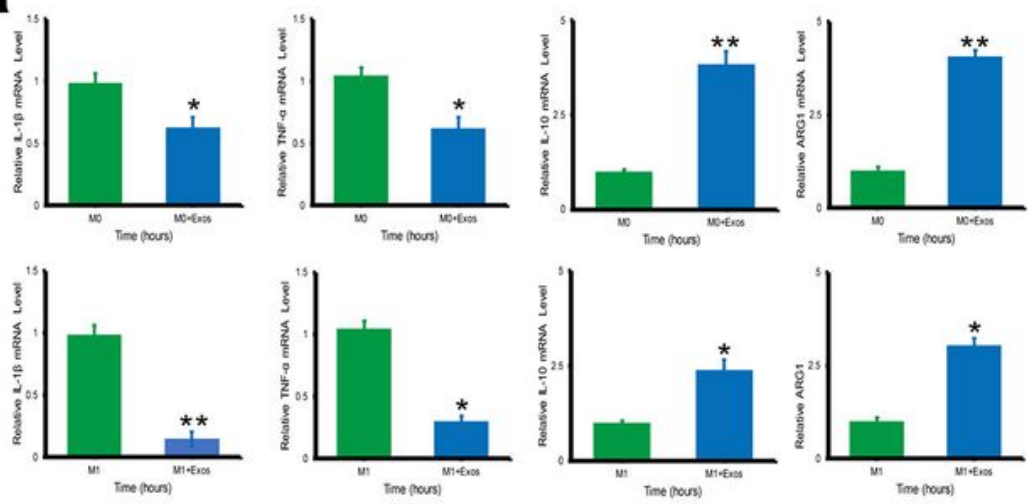

b

CD86
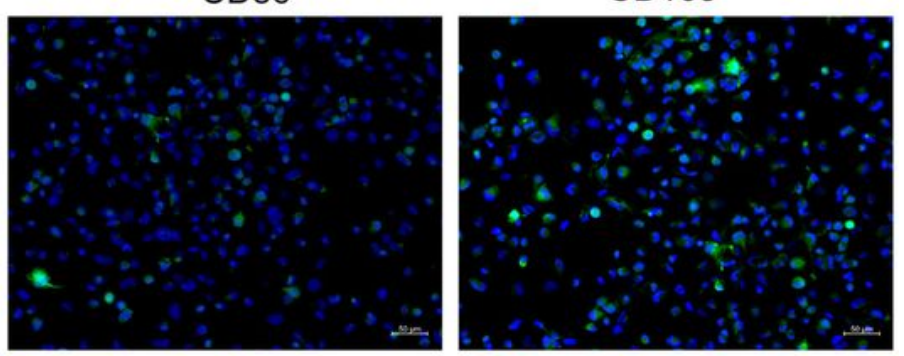

MO+Exos
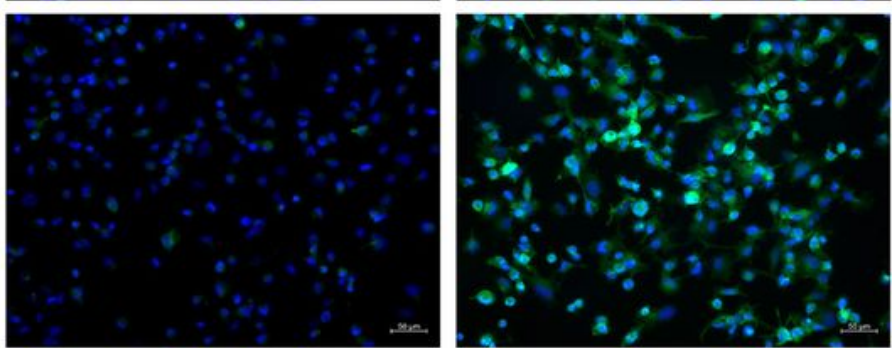

M1
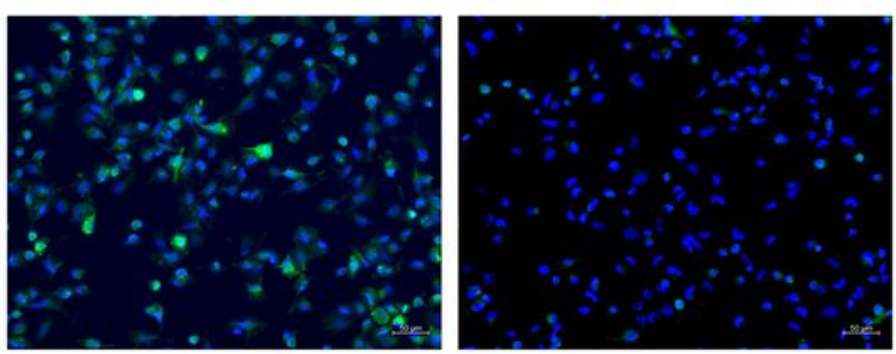

M1+Exos
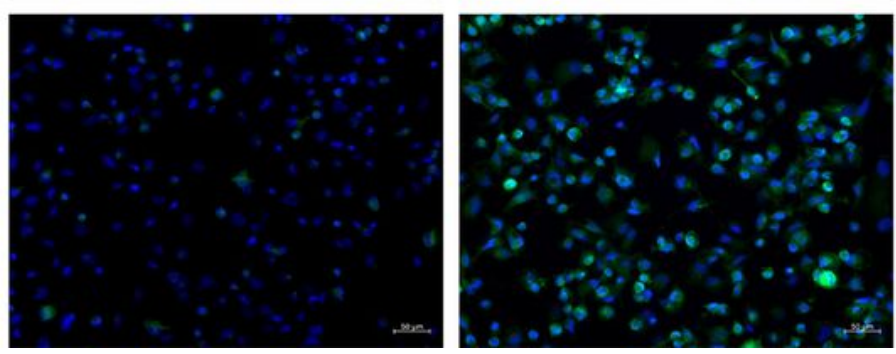

Figure 5 
Exosome effect on macrophage polarization. a Gene expression changes in macrophages of IL-1 $\beta$, TNFa, IL-10, ARG1 after stimulation with exosomes. This experiment was repeated three times. ${ }^{\star} \mathrm{P}<0.05$, $* * \mathrm{P}$ $<0.01$ compared to untreated macrophages. b CD86-, CD163-positive cells and representative images of fields showing green-colored cytoplasm of macrophages in each group. Hoechst 33342 staining was performed to detect nuclear localization (blue color). Scale bar: $100 \mu \mathrm{m}$.

a

ACLT+pMMx model

$1 \times 10^{* 0}$ hUC-MSCs-Exos on the first and

forth day of every week from the 5 th to

Harvest sample

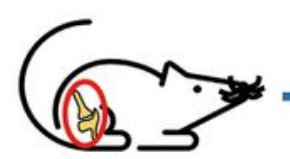

4 weeks

the 8th week
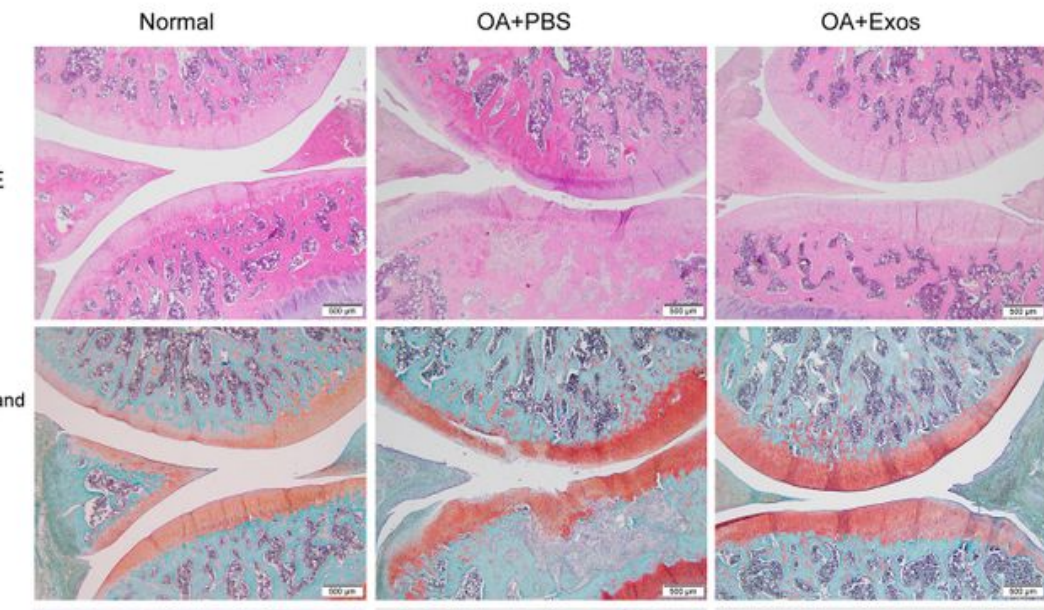

fast green
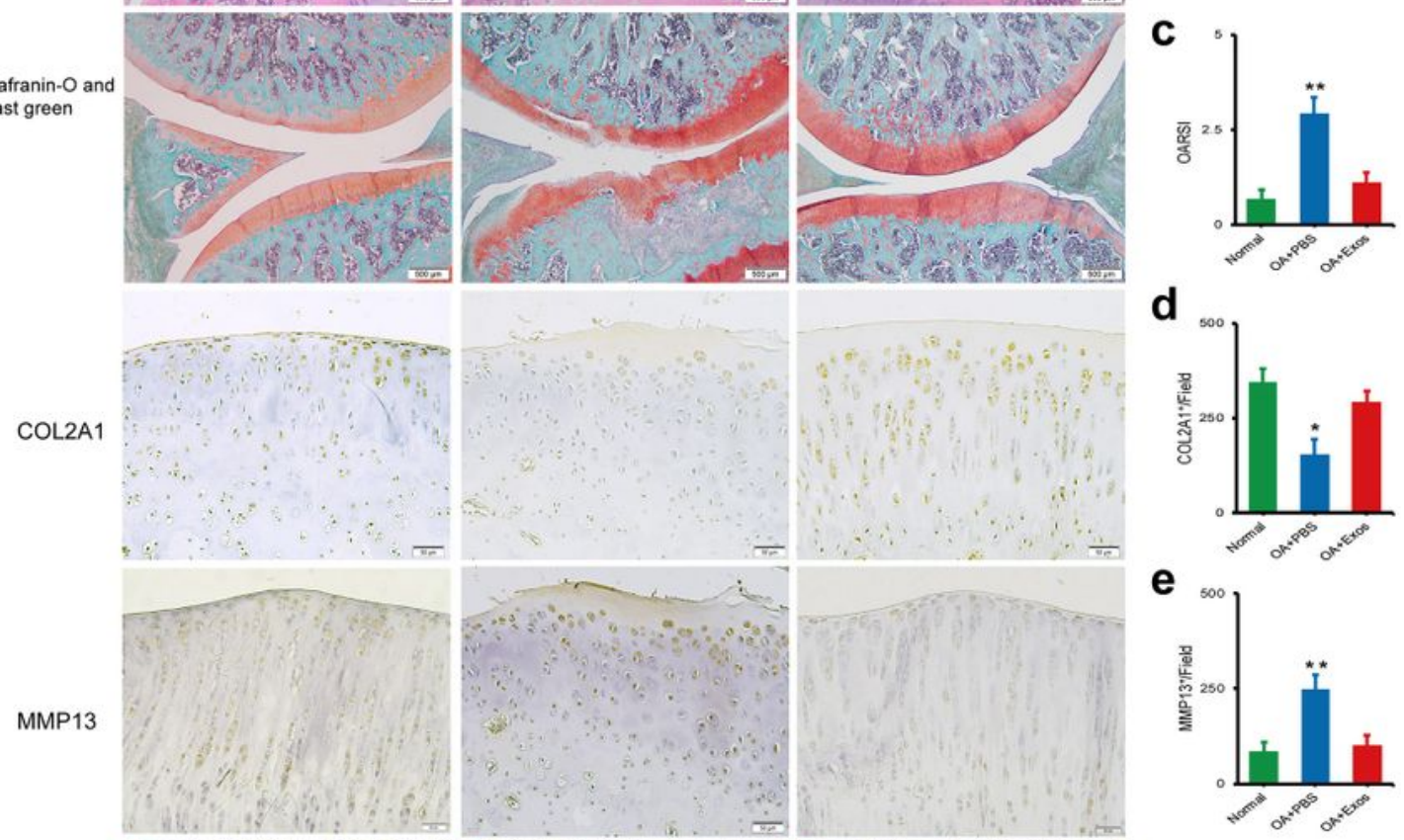

d

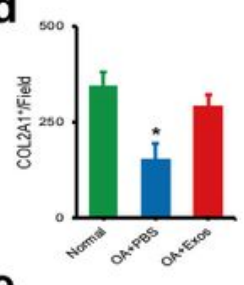

e

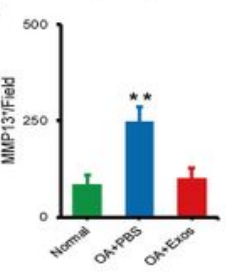

CD86
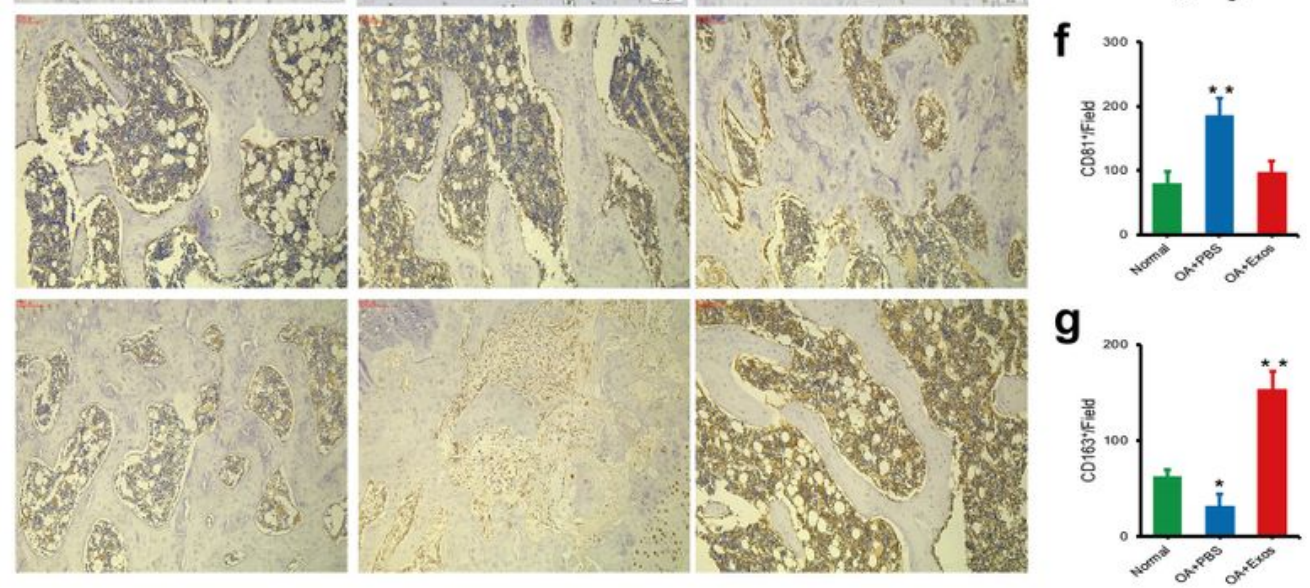

Figure 6 
hUC-MSCs-Exos prevent OA. a Flowchart of the in vivo experiment. b Staining results of hematoxylin and eosin (H\&E), safranin-O and fast green staining (Scale bar: $500 \mu \mathrm{m})$, and immunohistochemical staining for COL2A1, MMP-13 (Scale bar: $50 \mu \mathrm{m}$ ), CD86, and CD163 (Scale bar: $20 \mu \mathrm{m}$ ). c Statistical results of chondrocytes counted in randomly-selected fields and the result of statistical analysis of the osteoarthritis research society international (OARSI) score in each group. Data represent mean \pm SEM. $\star * P<0.01$ compared to the normal $(n=6)$. $d, e, f, g$ Statistical results of COL2A1+, MMP-13+, CD86+, CD163+ cells counted in randomly-selected fields. Data represent mean $\pm S E M$. $* P<0.05, * * P<0.01$ compared to the normal $(n=6)$.

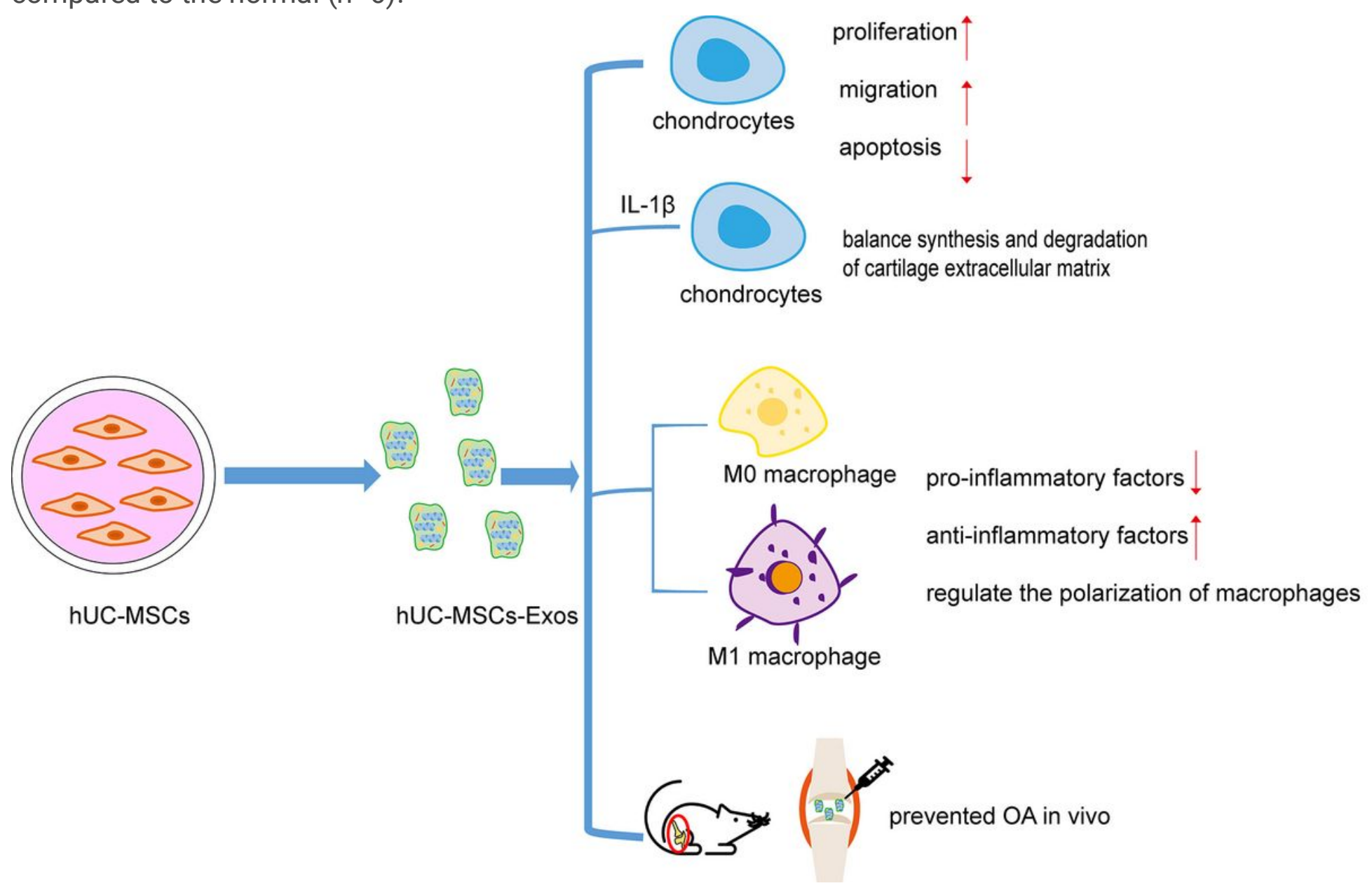

\section{Figure 7}

Graphical abstract of the experimental design. hUC-MSCs-Exos promote chondrocytes proliferation and migration and inhibit chondrocytes apoptosis. hUC-MSCs-Exos reversed IL-1 $\beta$-induced injury in vitro. hUCMSCs-Exos could inhibit the secretion of pro-inflammatory factors, promote the expression of antiinflammatory factors, and regulate the polarization of macrophages. hUC-MSCs-Exos attenuated the progression of $\mathrm{OA}$ and prevented severe damage to the knee articular cartilage in the rat $\mathrm{OA}$ model.

\section{Supplementary Files}

This is a list of supplementary files associated with this preprint. Click to download. 
- Certificateofediting.pdf

Page 29/29 